NASA Contractor Report 3437

\title{
On the Generation of Side-Edge Flap Noise
}

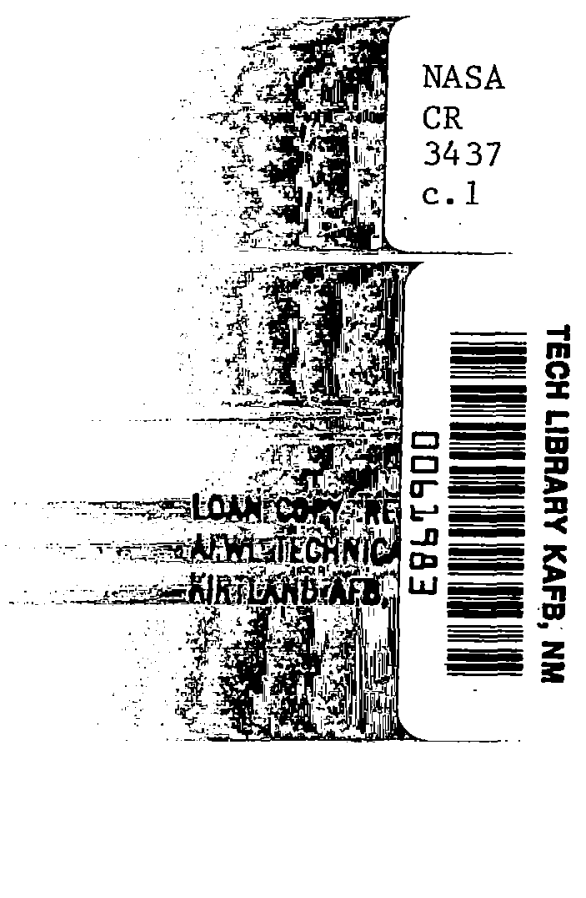

M. S. Howe

CONTRACT NAS1-16142

JUNE 1981 
NASA Contractor Report 3437

\section{On the Generation of Side-Edge Flap Noise}

M. S. Howe

Bolt Beranek and Newman Inc.

Cambridge, Massacbusetts

Prepared for

Langley Research Center

under Contract NAS1-16142

\section{N/SA}

National Aeronautics

and Space Administration

Scientific and Technical

Information Branch

1981 

TABLE OF CONTENTS

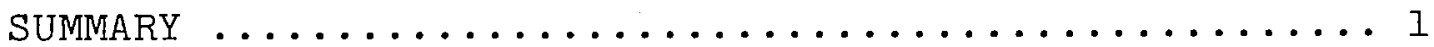

1. INTRODUCTION $\ldots \ldots \ldots \ldots \ldots \ldots \ldots \ldots \ldots \ldots \ldots \ldots \ldots \ldots$

2. FORMULATION OF THE AERODYNAMIC SOUND PROBLEM

IN TERMS OF DIFFRACTION THEORY $\ldots \ldots \ldots \ldots \ldots \ldots \ldots 3$

3. THE RADIATED SOUND $\ldots \ldots \ldots \ldots \ldots \ldots \ldots \ldots \ldots$

4. APPROXIMATE EVALUATION OF THE WAVENUMBER

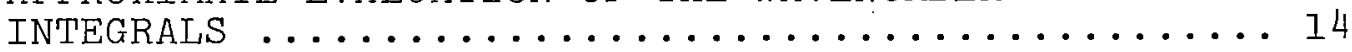

5. THE INTERRELATION BETWEEN THE SURFACE PRESSURE SPECTRUM AND THE ACOUSTIC SPECTRUM $\ldots \ldots \ldots \ldots \ldots \ldots$

6. COMPARISON WITH EXPERIMENT $\ldots \ldots \ldots \ldots \ldots \ldots \ldots \ldots$

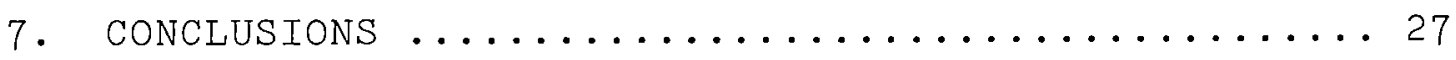

APPENDIX A. THE GREEN'S FUNCTION FOR A NONCOMPACT SLOT ............................. 29

APPENDIX B. THE GREEN'S FUNCTION FOR A COMPACT

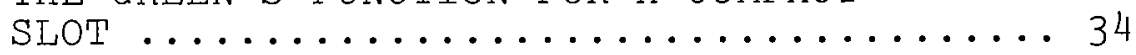

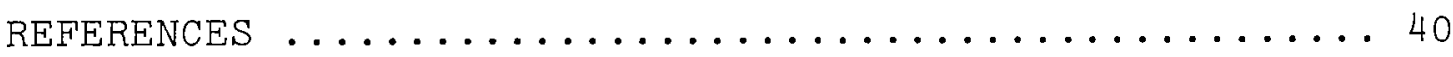




\section{LIST OF FIGURES}

Figure 1. (a) The "clean" trailing edge is represented analytically by means of a semiinfinite rigid plate in a mean flow at speed U. (b) The side-edge gap formed when a part-span flap of chord $L$ is deployed is modeled by a rectangular slot whose width $2 \mathrm{~s}$ is equal to the mean width of a side-edge gap.

(c) View of the slotted half-plane

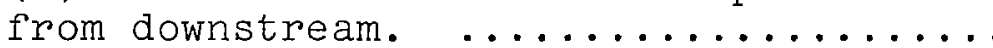

2. Illustrating the variation of the function $m(\lambda)$ defined in Appendix $B$ and used in the definition of the compact Green's function, for $2 \mathrm{~s} / \mathrm{L}=0.02,0.05,0.1 \ldots$

3. Variation of $\Delta(\Omega)$ (equation (39)) with Strouhal number $\Omega=\omega L / U$ in the flyover plane $\phi=0$, and for $\mathrm{M}=0.1, \mathrm{U}_{3} / \mathrm{U}=$ $0.2,2 \mathrm{~s} / \mathrm{L}=0.1 \ldots \ldots \ldots \ldots \ldots \ldots \ldots$

4. As for Fig. 3 with $M=0.3 \ldots \ldots \ldots .19$

5. As for Fig. 3 with $M=0.5 \ldots \ldots \ldots \ldots 20$

6. The field shape $\Delta(\Omega)$ (equation (39)) in the flyover plane $\phi=0$, for $\Omega=0.4$, 4,40 and for $M=0.1, U_{3} / U=0.2$,

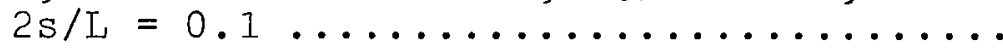

7. As for Fig. 6 with $M=0.3 \ldots \ldots \ldots \ldots 23$

8. As for Fig. 6 with $M=0.5 \ldots \ldots \ldots .24$ 


\section{SUMMARY}

A theory is proposed for estimating the noise generated at the side edges of part span trailing edge flaps in terms of pressure fluctuations measured just in-board of the side edge of the upper surface of the flap. Asymptotic formulae are developed in the opposite extremes of Lorentz contracted acoustic wavelength large/small compared with the chord of the flap. Interpolation between these limiting results enables the field shape and its dependence on subsonic forward flight speed to be predicted over the whole frequency range. It is shown that the mean width of the side edge gap between the flap and the undeflected portion of the airfoil has a significant influence on the intensity of the radiated sound. It is estimated that the noise generated at a single side edge of a full scale part span flap can exceed that produced along the whole of the trailing edge of the flap by 3 aB or more.

\section{INTRODUCTION}

A significant proportion of the noise generated by an aircraft on approach to landing is attributable to the deployment of high lift airframe components such as slats and flaps. Conventional jet-noise is of diminished importance in such circumstances because the engines are generally operated at reduced thrust. Fink and Schlinker [1], Ahtye, Miller and Meecham [2] and Kendall and Ahtye [3] have identified the side edges of part span trailing edge flaps as important sources of the airframe noise. In particular, the extrapolation to full scale of the experimental results of Kendall and Ahtye [3] leads to sound pressure levels which fall only a few $d B$ below flight data reported by Healy [4]. Hardin [5] has pointed out that the relative intensity of side edge sources could be substantially greater for future aircraft using high bypass ratio engines, which may well operate in the landing configuration with the jet stream impinging directly onto the side edges of flaps.

It is well known (Ffowes Williams and Hall [6]) that unsteady flow in the vicinity of an edge is an important source of aerodynamic sound. The generation of noise by turbulent flow over the trailing edge of an airfoil has been extensively discussed in the literature [7]. The studies cited above, however, suggest that for deployed part span flaps it is predominantly the characteristics of the unsteady flow in the vicinity of the side edges which determine the level and properties of the edge-generated sound. The unsteady 
motion in the neighborhood of a side edge is related to the presence of a component of mean flow about the edge. This flow results in the formation of a conventional tip vortex due to forward motion of a lifting airfoil, and is from the lower (pressure) to the upper (suction): surfaces of the flap, a separation bubble being formed on the upper surface at the edge. The relatively large amplitude unsteady edge flow is associated with the instability of this separated flow.

An idealized two-dimensional theory of the side-edge noise mechanism has been discussed by Hardin [5], who considered a flow inhomogeneity in the form of a chordwise orientated line vortex. Potential flow theory was used to model the mean flow about the side edge. By means of a detailed study of possible trajectories of the vortex, Hardin argued that under a wide range of conditions likely to be encountered in practice, the noise generated during the motion of the vortex around the side edge could be significantly greater than that produced by an equal (spanwise) vortex convecting in a mean boundary flow past a trailing edge. Such an idealized analysis can only be expected to yield possible trends, and, apart from considerations regarding the characterization of the unsteady edge flow, takes no account of important effects arising from the proximity of the wing ahead of the flap and of the side edges of the adjacent trailing edge of the wing. The latter are analogous to the so-called installation effects, which play an important role in dotcrmining the jet-noise of an aircraft (see $\operatorname{Ref.~8,9).~}$

In this report a theory is proposed which permits one to estimate the importance of proximity effects on the side-edge noise sources and, in addition, the influence of varying the forward flight speed of the aircraft. To do this the trailing edge of the wing is modeled by means of a thin, rigid halfplane in the "clean" configuration. When a part-span flap is deployed the gap between the side-edge of the flap and the undeflected portion of the neighboring trailing edge is represented by means of a narrow slot in the half-plane which is at right angles to the trailing edge. The length of this slot is equal to the chord of the flap, and the width is taken to be equal to the mean distance between the side edges of the flap and the adjacent undeflected wing (see Fig. I). The spanwise edge flow resulting from the additional lift generated by the flap is depicted schematically in Fig. I(c). In using this model to calculate the radiated sound, the finite angle of deflection of the flap is neglected, except insofar as it determines the magnitude of the mean flow velocity about the side edge. This approximation should be adequate for moderate angles of deflection, say of the order of $10^{\circ}$, in 
which case the spanwise component of the mean velocity on the upper surface close to the side edge is typically of order 0.2-0.3U, $U$ being the forward flight speed [10].

The analytical problem of calculating the sound generated by turbulent fluctuations in the spanwise flow near the side edge is formulated in 52 in terms of the evanescent wave theory of Chandiramani [11] and Chase $[12,13]$. This involves the use of the aeroacoustic Green's function whose normal. derivative vanishes on the slotted half-plane. Approximate representations of this Green's function in the opposite extremes. in which the chord of the flap is large/small relative to the Lorentz contracted acoustic wavelength are obtained in Appendices $A$ and $B$. These are used in $\$ 3$ to determine the spectrum of the acoustic radiation in terms of the wavenumberfrequency spectrum of the surface pressure fluctuations just in-board of the side edge of the flap. The introduction of further simplifying assumptions ( $\$ 4,5)$ enables the acoustic spectrum to be expressed directly in terms of a surface pressure point frequency spectrum. Interpolation between the low and high frequency limits in this case yields the approximate dependence of the radiation on all relevant Strouhal numbers (based on the chord of the flap) and forward flight Mach numbers. In $\$ 6$ a comparison is made with recent experimental results, and it is predicted that the noise generated at the side edge of a part span flap at full scale can exceed that generated along the whole of the trailing edge of the flap by up to $3 \mathrm{~dB}$.

\section{FORMULATION OF THE AERODYNAMIC SOUND PROBLEM IN TERMS OF DIFFRACTION THEORY}

A semi-infinite rigid plate which occupies the halfplane $\left(x_{1}<0, x_{2}=0\right)$ of a rectangular coordinate system $\left(x_{1}, x_{2}, x_{3}^{1}\right)$ is taken to model the trailing edge of the "clean" airfoil. The aerodynamic sound generated when a part-span flap is deployed will be calculated by introducing a rectangular slot of length $L$ and width $2 \mathrm{~s}$, with $2 \mathrm{~s}<\mathrm{L}$, to represent the side edge gap between the flap and the undeflected trailing edge. The slot occupies the region $\left(-\mathrm{L}<\mathrm{x}_{1}<0, \mathrm{x}_{2}=0,\left|\mathrm{x}_{3}\right|<\mathrm{s}\right)$ of the half-plane, as illustrated in Fig. I(b). The present analysis will take no account of the finite angle of deployment of the flap, although its influence is implicitly included in that it determines the magnitude of.the mean flow about the side edge and the associated turbulence levels. In addition, the possible existence of a spanwise slot ahead of the flap [indicated by the dashed lines in Figs. I(a), I(b)] is ignored, as is the small finite extension of the flap beyond the nominal 

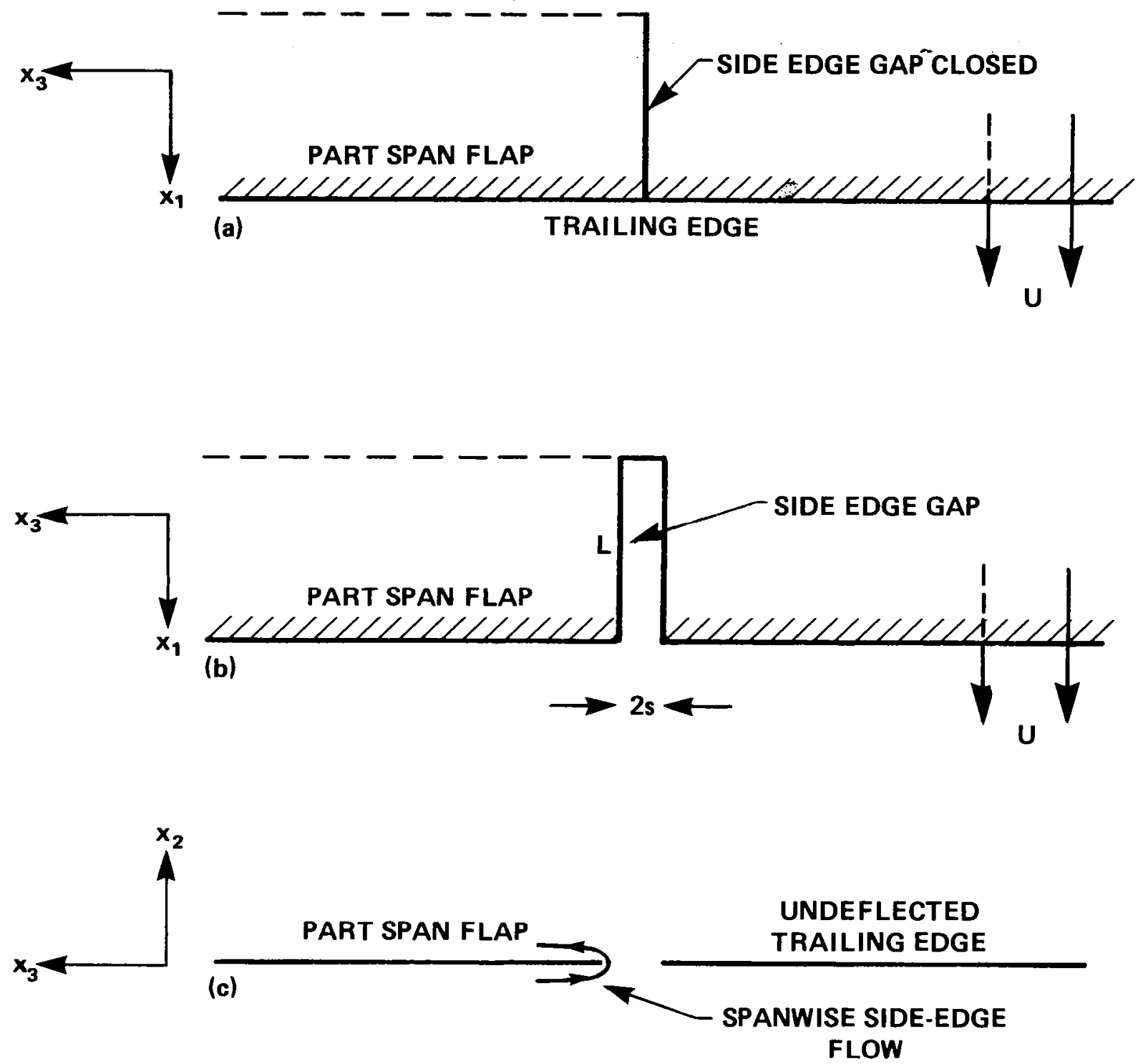

FIG. 1. (a) THE "CLEAN" TRAILING EDGE IS REPRESENTED ANALYTICALLY BY MEANS OF A SEMI-INFINITE RIGID PLATE IN A MEAN FLOW AT SPEED $U$. (b) THE SIDE-EDGE GAP FORMED WHEN A PART-SPAN FLAP OF CHORD L IS DEPLOYED IS MODELED BY A RECTANGULAR SLOT WHOSE WIDTH $2 S$ IS EQUAL TO THE MEAN WIDTH OF A SIDE-EDGE GAP. (c) VIEW OF THE SLOTTED HALF-PLANE FROM DOWNSTREAM. IN CALCULATING THE NOISE THE FINITE ANGLE OF DEPLOYMENT OF THE FLAP IS NEGLECTED. THE FLAP OCCUPIES THE REGION $\left(-L<x_{1}<0, x_{2}=0, x_{3}>S\right)$. 
trailing edge $\left(x_{1}=x_{2}=0\right)$ of the wing. Forward flight is represented by a uniform mean flow on both sides of the airfoil at subsonic speed $U$ in the positive direction of the $x_{1}$-axis.

The instability of the separated spanwise flow over the upper surface of the flap near the side edge gives rise to turbulent surface pressure fluctuations whose magnitudes in the immediate vicinity of the edge are influenced by the presence of the slot. It is convenient to characterize these surface pressures in terms of an hypothetical surface pressure $P\left(x_{1}, x_{3}, t\right)$ say, that the same turbulence would induce on a plane rigid wall lying in $x_{2}=0$. If it could be assumed that the turbulence convects in a frozen pattern over the flap, $P$ would be equal to the surface pressure fluctuation at distances in-board from the edge which exceed the correlation scale of the turbulence. The formulation of the noise problem in terms of $P$ is equivalent to the use of "evanescent wave theory" in the calculation of trailing edge noise [11-13]. The principal advantage in the use of $P$ is that it may be assumed to possess local spatial stationarity in the spanwise direction, a property not shared by the actual surface pressure close to the side edge. In practice, surface pressure measurements in-board of the edge would have to be used to quantify turbulence decay, etc., and thereby to estimate an approximate form of $P$ near the edge (c.f., the analogous measurements performed by Brooks and Hodgson [14]).

Consider an elementary turbulent boundary layer disturbance above the flap (in $x_{2}>0$ ) which is proportional to $\exp \left\{i\left(k_{1} x_{1}+k_{3} x_{3}-w t\right)\right\}$. Henceforth, we shall omit from our formulae explicit reference to the exponential time factor $e^{-i \omega t}$. Practically all of the turbulence energy will be associated with disturbances having subsonic phase velocities in the $\left(x_{1}-x_{3}\right)$-plane. Hence, if the flap and airfoil were absent, the corresponding perturbation pressure would decay exponentially with perpendicular distance below the boundary layer, and would form an evanescent wave whose pressure signature PI could be expressed as

$$
p_{I}=\hat{p}_{I}\left(k_{1}, k_{3}, \omega\right) e^{i\left(k_{1} x_{1}+k_{3} x_{3}\right)+\Gamma x_{2}}
$$

where $\Gamma=\Gamma\left(k_{1}, k_{3}, \omega\right)>0$. The functional form of $\Gamma$ will be discussed below. 
Just above the flap, at distances from the side and trailing. edges which exceed $\sim I /\left(k_{1}^{2}+k_{3}^{2}\right)^{\frac{1}{2}}$, the harmonic disturbance ( 1 ) gives rise to a specularly reflected pressure field such that the net surface pressure $P\left(x_{1}, x_{2}, t\right)$ is given by

$$
P=\hat{P}\left(k_{1}, k_{3}, \omega\right) e^{i\left(k_{1} x_{1}+k_{3} x_{3}\right)},
$$

where,

$$
\hat{\mathrm{P}}=2 \hat{\mathrm{p}}_{I} \text {. }
$$

Sound waves are generated when the evanescent wave ( 1 ) is diffracted by inhomogeneities such as the edges of the flap. The side-edge flap noise corresponds to the diffraction radiation produced at the slot, and use of $\mathrm{Eq}$. (3) in the diffraction calculation enables the acoustic pressure to be related directly to the surface pressure on the flap in-board of the side edge.

The diffraction problem is formulated as follows. Let $p_{S}$ denote the diffracted component of the perturbation pressure. The net pressure is accordingly given by

$$
p=p_{I}+p_{S}
$$

and is such that on the upper surface of the flap

$$
p_{S} \rightarrow p_{I}, p \rightarrow P
$$

at large distances from the slot and trailing edge. At large distances from the airfoil, $p_{S}$ represents the acoustic radiation. In addition, $p_{S}$ must be chosen to ensure that the normal velocity vanishes on the surface of the airfoil. In the high Reynolds number limit which is relevant in practice, unsteady viscous stresses may be neglected, and the $\mathrm{x}_{2}$-component of the momentum equation implies that the normal component of velocity will vanish on the airfoil provided that 


$$
\partial p_{I} / \partial x_{2}+\partial p_{S} / \partial x_{2}=0
$$

on the upper and lower surfaces of the slotted half-plane.

Acoustic disturbances are convected by mean flow. Since, however, the nonuniform mean flow in the vicinity of the slot is localized, its effect on propagation will be neglected, and in the linearized approximation it therefore follows that $\mathrm{p}_{\mathrm{s}}$ satisfies the time-harmonic convected wave equation:

$$
\left\{\left(-i k_{0}+\frac{M \partial}{\partial x_{1}}\right)^{2}-\frac{\partial^{2}}{\partial x_{j}^{2}}\right\} p_{S}(\underline{x}, \omega)=0,
$$

where $M=U / c<I$ is the mean flow (flight) Mach number, $\mathrm{k}_{0}=\omega / \mathrm{c}$ the acoustic wavenumber, and $\mathrm{c}$ is the speed of sound. The solution which satisfies the radiation condition of outgoing waves at large distances together with the surface condilion (5) can be written down with the aid of a Green's function $G(x, y)$. This satisfies $\partial G / \partial x_{2}=0$ on the slotted half-plane, the radiation condition, and equation ( 6 ) when the right hand side is replaced by the point source $\delta(x-y)$. According to the reverse-flow reciprocal theorem [15], G(x,y) is also a solution as a function of $y$ of the adjoint or reverse flow problem:

$$
\left\{\left(-i k_{0}-\frac{M \partial}{\partial y_{1}}\right)^{2}-\frac{\partial^{2}}{\partial y_{j}^{2}}\right\} G(\underline{x}, \underline{y})=\delta(\underline{x}-\underline{y}),
$$

and satisfies the radiation condition and $\partial G / \partial y_{2}=0$ on the slotted half-plane. In the reciprocal problem the source $\delta(\underline{x}-\underline{y})$ is situated at the observer position $x$ and generates a disturbance which propagates in $\mathrm{y}$-space in a medium moving at speed $\mathrm{U}$ in the negative $\mathrm{y}_{1}$-direction.

A formal representation of the radiated sound is obtained by means of Kirchhoff's integration procedure ([16], Chap. 8). In equation (6) replace the dummy variable $x$ by $y$, multiply by $G(\underline{x}, \underline{y})$ and integrate with respect to $\underline{y}$ over the region of space bounded by the airfoil and a large sphere of radius $R^{*}$, say. Similarly, multiply Eq. (7) by $p_{S}(y)$ and integrate over the same region. Subtract the two results and apply the divergence theorem to obtain, as $\mathrm{R}^{*} \rightarrow \infty$ : 


$$
p_{S}(\underline{x}, \omega)=\oint_{S_{ \pm}} G(\underline{x}, \underline{y}) \cdot \frac{\partial p_{S}}{\partial y_{n}}(\underline{y}) d y_{1} d y_{3}
$$

where the integration is over the upper and lower surfaces $\mathrm{S}_{+}$respectively of the slotted half-plane, and $\mathrm{y}_{\mathrm{n}}$ is in the direction of the outward normal. In obtaining this expression the radiation condition has been used to eliminate contributions from the surface of the sphere, and use has been made of $\partial G / \partial y_{2}=0$ on the rigid surface of the airfoil. Equation (8) is expressed in terms of the incident, evanescent wave $p_{I}$ by means of equation (5), expressed in the form

$$
\partial p_{s} / \partial y_{n}=\mp \partial p_{I} / \partial y_{2}
$$

on $S_{ \pm} \cdot$ If we next define

$$
G_{ \pm}(\underline{x})=G\left(\underline{x}, y_{1}, \pm 0, y_{3}\right),
$$

equation ( 8 ) becomes:

$$
p_{S}(\underline{x}, \omega)=-\int_{S_{+}}\left[G_{+}(\underline{x})-G_{-}(\underline{x})\right] \frac{\partial p_{I}}{\partial y_{2}}(\underline{y}) d y_{1} d y_{3}
$$

the integration being over that portion of the upper surface of the slotted airfoil wherein $\partial \mathrm{p}_{I} / \partial \mathrm{y}_{2}$ is different from zero. Using equations (I), (3) and summing over all possible values of the wavenumbers $k_{1}, k_{3}$, the radiation is expressed, finally, in terms of the surface pressure amplitude coefficient $\hat{\mathrm{P}}\left(\mathrm{k}_{1}, \mathrm{k}_{3}, \omega\right)$ :

$p_{S}(\underline{x}, w)=$

$-\frac{1}{2} \iint_{\infty}^{\infty} d k_{1} d k_{3} \Gamma\left(k_{1}, k_{3}, \omega\right) \hat{P}\left(k_{1}, k_{3}, \omega\right) \int_{S_{+}}\left[G_{+}(\underline{x})-G_{-}(\underline{x})\right] e^{i\left(k_{1} y_{1}+k_{3} y_{3}\right)} d y_{1} d y_{3}$ 
Equation (12) may be used to express the frequency spectrum $S(x, \omega)$, say, of the acoustic radiation in terms of the wavenumber-frequency spectrum $\pi\left(k_{1}, k_{3}, \omega\right)$ of the surface pressure $P\left(x_{1}, x_{3}, t\right)$. We recall that these quantities are defined through the relations:

$$
\begin{gathered}
<p_{S}(\underline{x}, t) p_{S}(\underline{x}, t+\tau)>=\int_{-\infty}^{\infty} S(\underline{x}, \omega) e^{-i \omega \tau} d \omega, \\
\left\langle P\left(y_{1}, y_{3}, t\right) P\left(y_{1}+\bar{y}_{1}, y_{3}+\bar{y}_{3}, t+\tau\right)>\right. \\
=\iiint_{-\infty}^{\infty} \pi\left(k_{1}, k_{3}, \omega\right) e^{i\left(k_{1} \bar{y}_{1}+k_{3} \bar{y}_{3}-\omega \tau\right)} d k_{1} d k_{3} d \omega,
\end{gathered}
$$

in which the angle brackets denote an ensemble average. Introducing the hypothesis that $P\left(y_{1}, y_{3}, t\right)$ is locally a stationary random function of its arguments, with zero mean (a reasonable assumption inasmuch as $\mathrm{P}$ is defined independently of the diffracted field), it follows that:

$$
\begin{aligned}
& \left.<\hat{\mathrm{P}}\left(\mathrm{k}_{1}, \mathrm{k}_{3}, \omega\right) \hat{\mathrm{P}}\left(\overline{\mathrm{k}}_{1}, \overline{\mathrm{k}}_{3}, \bar{\omega}\right)\right\rangle \\
& =\delta\left(\mathrm{k}_{1}+\overline{\mathrm{k}}_{1}\right) \delta\left(\mathrm{k}_{3}+\overline{\mathrm{k}}_{3}\right) \delta(\omega+\bar{\omega}) \Pi\left(\mathrm{k}_{1}, \mathrm{k}_{3}, \omega\right),
\end{aligned}
$$

and similarly that:

$$
\left\langle\mathrm{p}_{\mathrm{S}}(\underline{\mathrm{x}}, \omega) \mathrm{p}_{\mathrm{S}}(\underline{\mathrm{x}}, \bar{\omega})\right\rangle=\delta(\omega+\bar{\omega}) \mathrm{S}(\underline{\mathrm{x}}, \omega) .
$$

These results will be used below.

\section{THE RADIATED SOUND}

The practical implementation of the formal solution (12) requires a knowledge of the Green's function $G(x, y)$. Approximate analytical representations of $G$ are availabie in the two asymptotic extremes in which the chord $L$ of the flap satisfies

$$
k_{0} L /\left(1-\mathbb{M}^{2}\right)>>,<1 .
$$


The details of the calculation of $G$ in these two cases are outlined in the Appendices under the additional restriction that $k_{0} s \ll I$, i.e., that the acoustic wavelength is large compared with the side edge gap.

Attention will be confined to the side-edge noise problem in which $\mathrm{P}\left(\mathrm{y}_{1}, \mathrm{y}_{3}, t\right)$ may be taken to be nonzero only in the immediate vicinity of the slot on the upper surface of the flap $\left(-\mathrm{L}<\mathrm{y}_{1}<0, \mathrm{y}_{2}=+0, \mathrm{y}_{3}>\mathrm{s}\right)$. The noncompact slot $\left(\mathrm{k}_{0} \mathrm{I}>>\mathrm{I}\right)$ is discussed in Appendix ${ }^{3}$, and it follows from Eq. (A20) that, when the observer is in the far f'ield, and $y_{3}>5$ (on the upper surface of the flap),

$$
G_{+}(\underline{x})-G_{-}(\underline{x})=\frac{F_{N C} e^{i k_{0} r}}{r} \cdot \ln \left[\frac{y_{3}}{s}+\sqrt{\frac{y_{3}^{2}}{s^{2}}-1}\right],
$$

where

$$
F_{N C}=\frac{-i}{\pi^{2}(I+M \cos \theta)\left\{1+\frac{2 i}{\pi} \cdot \ln \left[C k_{0} s \cdot \sin \theta / 4(I+M \cos \theta)\right]\right\}}
$$

and $\mathrm{C}=1.78107 \ldots$ is the Euler-Mascheroni constant. In these expressions the observer position relative to the airfoil is specified in a form appropriate to aircraft flight at speed $U$ in the negative $x_{1}$-direction by spherical polar coordinates $(r, \theta, \phi)$ at the time of emission of the received sound, where the polar angle $\theta$ is measured from the positive direction of the $x_{\text {taxis }}$ [see Eqs. (A.18) of Appendix A]. The emissiontime distance $r$ is given by

$$
r=\frac{1}{\left(1-\mathbb{M}^{2}\right)^{\frac{1}{2}}}\left\{\frac{-\mathbb{M}\left(x_{1}-y_{1}\right)}{\left(1-M^{2}\right)^{\frac{1}{2}}}+\sqrt{\frac{\left(x_{1}-y_{1}\right)^{2}}{1-M^{2}}+x_{2}^{2}+x_{3}^{2}}\right\} .
$$


Substituting from (18) into Eq. (12), we find, as $k_{0} r+\infty$ :

$p_{S}(\underline{x}, \omega) \simeq$

$\iint_{-\infty}^{\infty} d k_{1} d k_{3} \frac{\Gamma \hat{P} F}{2 r_{0}} \int_{-L}^{0} e^{i\left(k_{0} r+k_{1} y_{1}\right)} d y_{1} \int_{S}^{\infty} e^{i k_{3} y_{3}} \ln \left[\frac{y_{3}}{s}+\sqrt{\frac{y_{3}^{2}}{s^{2}}-I}\right] d y_{3}$.

, (21)

in which the spanwise integration has been extended to $\mathrm{y}_{3}=+\infty$ since in practice the span of the flap will be large compared with the characteristic turbulence scale. Convergence of the integral in this limit is ensured by assigning to $k_{3}$ a small positive imaginary component which is subsequently allowed to vanish. The distance $r_{0}$ is defined by Eq. (20) when $\mathrm{y}_{1}=0$, and to leading order we have:

$$
r=r_{0}-y_{1} \cos \theta /(1+M)(1+M \cos \theta),
$$

where use has been made of the relations (Al8). It follows from this and the integral definition of the Hankel function $\mathrm{H}_{0}^{(1)}(z)$ ([17], page 955), that

$p_{S}(\underline{x}, \omega) \simeq \iint_{-\infty}^{\infty} \frac{\pi \hat{P} F_{N C} H_{0}^{(1)}\left(k_{3} s\right)}{2 k_{3} r_{0}} \cdot \frac{\sin \left\{\frac{1}{2} L\left[k_{1}-k_{0} \cos \theta /(1+M)(1+M \cos \theta)\right]\right\}}{\left[k_{1}-k_{0} \cos \theta /(1+M)(1+M \cos \theta)\right]}$

$\times \exp \left\{i\left[k_{0} r_{0}-\frac{I}{2} L\left(k_{1}-k_{0} \cos \theta /(1+M)(1+M \cos \theta)\right)\right]\right\} d k_{1} d k_{3}$.

Let $\mathrm{S}_{\mathrm{NC}}(\omega)$ denote the frequency spectrum of the acoustic pressure in the noncompact limit, defined as in Eq. (16). Equation (23) can be used to form the product on the left of (16) which, used in conjunction with Eq. (15), yields: 


$$
\begin{aligned}
S_{N C}(\omega) \simeq & \frac{\pi^{2}\left|F_{N C}\right|^{2}}{4 r_{0}^{2}} \iint_{\infty}^{\infty}\left|\frac{\Gamma H_{0}^{(I)}\left(k_{3} s\right)}{k_{3}}\right|^{2} \pi\left(k_{1}, k_{3}, \omega\right) \times \\
& \times \frac{\sin ^{2}\left\{\frac{1}{2} L\left[k_{1}-k_{0} \cos \theta /(I+M)(I+M \cos \theta)\right]\right\}}{\left\{k_{1}-k_{0} \cos \theta /(I+M)(I+M \cos \theta)\right\}^{2}} d k_{1} d k_{3} .
\end{aligned}
$$

The formulae given in Appendix B for the Green's function for a compact slot $\left[\mathrm{k}_{0} \mathrm{~L} /\left(\mathrm{l}-\mathrm{M}^{2}\right)<1\right]$ may be used similarly to calculate the corresponding acoustic pressure spectrum $\mathrm{S}_{\mathrm{C}}(\omega)$, say. One finds in this case:

$S_{C}(\omega) \simeq$

$$
\frac{\pi^{2}}{I 6}\left(\frac{L}{r_{0}}\right)^{2}\left|F_{C}\right|^{2} \iint_{-\infty}^{\infty}\left|\frac{\Gamma f\left(k_{1} L\right) H_{0}^{(I)}\left(k_{3} s\right)}{k_{3}}\right|^{2} \pi\left(k_{1}, k_{3}, \omega\right) d k_{1} d k_{3},(25)
$$

in which

$$
\begin{aligned}
& F_{C}=\left[\frac{2 k_{0} L\left\{(M+\cos \theta)^{2}+\left(1-M^{2}\right) \cos ^{2} \phi \sin ^{2} \theta\right\}^{\frac{1}{2}}}{i \pi\left(1-M^{2}\right)(1+M \cos \theta)}\right]^{\frac{1}{2}} \frac{\sin \left(\frac{1}{2} \bar{\theta}\right)}{\pi(I+M \cos \theta)}, \\
& f(z)=\int_{0}^{1} m(\lambda) e^{-i z \lambda} d \lambda .
\end{aligned}
$$

The function $m(\lambda)(0<\lambda<1)$ is defined in Appendix $B$, and depends on the value of the slot width/length ratio $2 \mathrm{~s} / \mathrm{L}$. Its behavior for $2 \mathrm{~s} / \mathrm{L}=0.02,0.05,0.1$ is illustrated in Fig. 2. The angle $\bar{\theta}$ is given in terms of $(\theta, \phi)$ by $\mathrm{Eq} \cdot(\mathrm{BI})$. When $\phi=0$, i.e., in the "flyover" plane, we have

$$
\sin \left(\frac{1}{2} \bar{\theta}\right)=\sin \left(\frac{1}{2} \theta\right)\{(1-M) /(1+M \cos \theta)\}^{\frac{1}{2}} .
$$




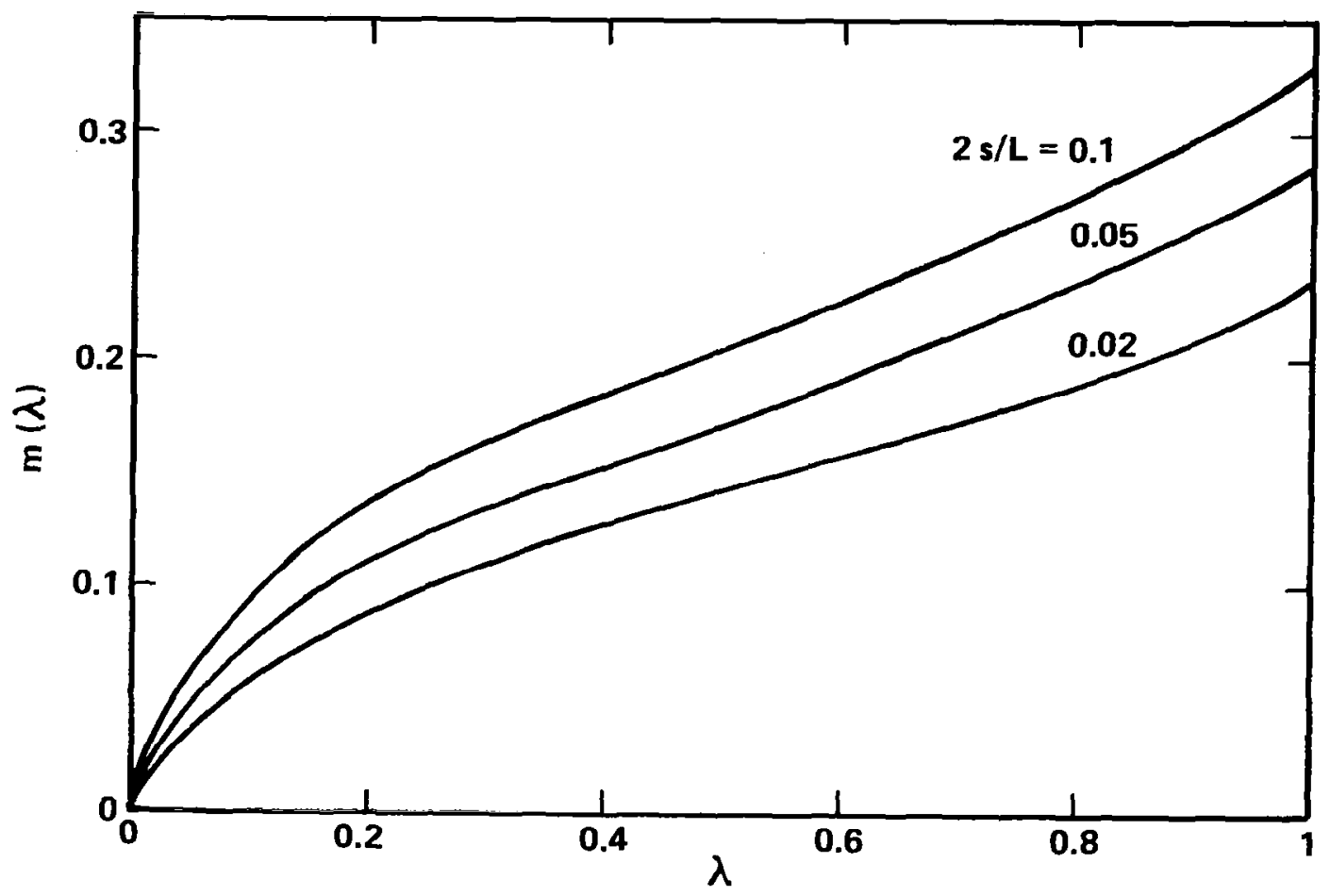

FIG. 2. ILLUSTRATING THE VARIATION OF THE FUNCTION $m(\lambda)$ DEFINED IN APPENDIX B AND USED IN THE DEFINITION OF THE COMPACT GREEN'S FUNCTION, FOR $2 \mathrm{~s} / \mathrm{L}=0.02,0.05,0.1$. 
It should be noted that Eqs. (24), (25) specify the acoustic spectrum in terms of the frequency $\omega$ as measured by an observer fixed relative to the airfoil. This is related to the frequency $\omega^{\prime}$ that would be measured by an observer at rest relative to the mean flow by the usual Doppler relation $\omega^{\prime}=\omega /(i+M \cos \theta)$.

\section{APPROXIMATE EVALUATION OF THE WAVENUMBER INTEGRALS}

The integrals in Eqs. (24), (25) can be estimated by first noting that, in the region in which the surface pressure spectrum $I\left(k_{1}, k_{3}, \omega\right)$ is significantly different from zero, $k_{1} L \gg I$ ( $i . e$. , turbulence scales are small compared with the chord of the flap), and therefore that the sine in Eq. (24) and $f\left(k_{1} L\right)$ in (25) will vary rapidly with $k_{1}$. In the case of Eq. (24), this implies that the principal contribution to the $k_{1}$-integral is" from the neighborhood of

$$
k_{1}^{*}=k_{0} \cos \theta /(1+M)(1+M \cos \theta) \text {. }
$$

At this relatively small value of $k$ the surface pressure spectrum is likely to be sharply peaked near $k_{3}=\omega / U_{3}$, where $\mathrm{U}_{3}$ is a velocity which is of the same order as the mean spanwise velocity component near the side edge. The value of the $\mathrm{k}_{3}$-integral in (24) may accordingly be approximated by setting $\mathrm{k}_{3}=\omega / \mathrm{U}_{3}$ in the integrand except in the argument of $\pi$. Our approximate representation of $\mathrm{S}_{\mathrm{NC}}(\omega)$ is therefore:

$$
\begin{aligned}
S_{N C}(\omega) \simeq & \frac{\pi^{3} \mathrm{~L}}{8 r_{0}^{2}}\left|F_{N C}\right|^{2}\left|\frac{\Gamma\left(k_{1}^{*}, \omega / U_{3}, \omega\right) H_{0}^{(I)}\left(\omega s / U_{3}\right)}{\omega / U_{3}}\right|^{2} \\
& \times \int_{-\infty}^{\infty} \Pi\left(k_{1}^{*}, k_{3}, \omega\right) \mathrm{dk}_{3} .
\end{aligned}
$$

Further reduction of this result is achieved by observing that, if it can be assumed that the evanescent wave ( 1 ) is convected by the mean flow in the same way as acoustic waves, we have

$$
\Gamma=\left\{k_{1}^{2}+k_{3}^{2}-\left(k_{0}-I M k_{1}\right)^{2}\right\}^{\frac{3}{2}} .
$$


But $\mathrm{M}_{3}=\mathrm{U}_{3} / \mathrm{c} \sim 0.2 \mathrm{M}$ (see Ref. 10) for moderate subsonic mean flow Mach numbers $M$. It follows that $M^{2}$ is generally negligible compared with unity, and when this is used in (31) with $k_{1}=k_{1}^{*}$, it can be seen that in (30) we may take

$$
\Gamma=\left|k_{3}\right|=|\omega| / U_{3} \text {. }
$$

It may be remarked that this approximation indicates that the possible uncertainty in the precise value of the convection velocity to be used in the definition of $\Gamma$ is unlikely to be significant.

Similarly, $k_{1}^{*}$ may be replaced by zero in the integrand of Eq. (30), since for small spanwise Mach number $M_{3}$ the acoustic wavelength will greatly exceed the characteristic turbulence scale. This approximation permits the introduction of the point surface spectrum $\Phi(\omega)$, defined by

$$
\ell_{1} \Phi(\omega)=\int_{-\infty}^{\infty} \pi\left(0, k_{3}, \omega\right) d k_{3},
$$

where $\ell \sim_{3} / \omega$ is the characteristic turbulence integral scale in the mean flow direction. This defines the local mean square surface pressure $P\left(x_{1}, x_{3}, t\right)$ through:

$$
\left\langle\mathrm{P}^{2}\right\rangle=\int_{-\infty}^{\infty} \Phi(\omega) \mathrm{d} \omega
$$

The use of Eq. (32), (33) in Eq. (30) gives our final approximation for the acoustic spectrum in the noncompact limit:

$$
S_{N C}(\omega) \simeq \frac{\pi^{3}}{8}\left(\frac{\ell_{1} L}{r_{0}^{2}}\right)\left|F_{N C}\right|^{2}\left|H_{0}^{(I)}\left(\omega S / U_{3}\right)\right|^{2} \Phi(\omega) .
$$

Introducing the same approximations into the evaluation of the wavenumber integrals in $\mathrm{Eq}$. (25), one obtains in the compact limit: 


$$
S_{C}(\omega) \simeq \frac{\pi^{3}}{8}\left(\frac{l_{1} L}{r_{0}^{2}}\right)\left|F_{C}\right|^{2}\left|H_{0}^{(I)}\left(\omega s / U_{3}\right)\right|^{2} I(2 s / L) \Phi(\omega),
$$

in which

$$
I=\int_{0}^{1}|m(\lambda)|^{2} \mathrm{~d} \lambda
$$

This integral is a function of the slot width/length ratio $2 \mathrm{~s} / \mathrm{L}$, and typical values are given in Table 1 :

TABLE 1

\begin{tabular}{|c|l|l|l|}
\hline $2 \mathrm{~S} / \mathrm{I}$ & 0.02 & 0.05 & 0.1 \\
\hline$I$ & 0.022 & 0.033 & 0.046 \\
\hline
\end{tabular}

5. THE INTERRELATION BETWEEN THE SURFACE PRESSURE SPECTRUM AND THE ACOUSTIC SPECTRUM

Equations (35), (36) provide an approximate relation between the acoustic frequency spectrum $S(\omega)$ and the point surface pressure spectrum $\Phi(\omega)$, although in practice $\Phi(\omega)$ should perhaps be regarded as a chordwise averaged spectrum. The chordwise correlation scale $l$ is of order $U / \omega$. To exhibit an explicit relationship between $S(\omega)$ and $\Phi(\omega)$ we shall write

$$
\ell_{1}=\alpha U / \omega,
$$

In which $\alpha$ is taken to be constant in the region in which $\Phi(\omega)$ is significantly different from zero. In order of magnitude it might be anticipated that $\alpha \sim U_{3} / U$, although its precise value will not be required in this section.

The quantity

$$
\Delta(\omega)=10 . \log _{10}\left\{S(\omega) / \alpha \Phi(\omega)\left(L / r_{0}\right)^{2}\right\},
$$


gives the relation in $\mathrm{dB}$ between the sound pressure level at frequency $\omega$ and the surface pressure spectrum. We now proceed to discuss the dependence of $\Delta(\omega)$ on the chordwise Strouhal number $\Omega=\omega \mathrm{L} / \mathrm{U}$ for radiation directions which lie in the flyover plane $\phi=0$.

From Eq. (35) it is seen that the noncompact component $\Delta_{\mathrm{NC}}(\Omega)$ is given by

$\Delta_{\mathrm{NC}}(\Omega)$

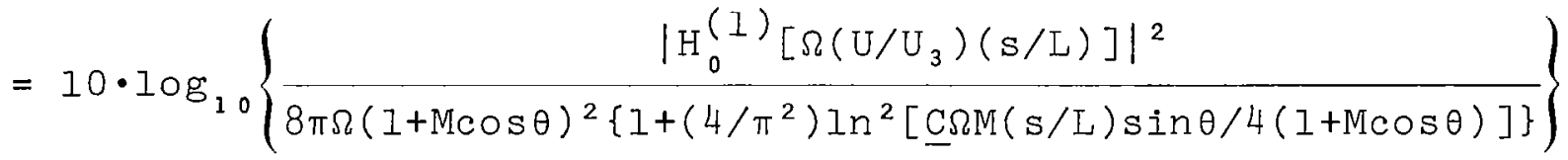

The noncompact approximation requires for its validity that $\omega \mathrm{L} / \mathrm{c}>1, \omega \mathrm{s} / \mathrm{c}<1$, so that the outer limits of validity of $\mathrm{Eq}$. (40) are delimited by

$$
I / \mathbb{M}<\Omega<(\mathrm{L} / \mathrm{S}) / \mathrm{M}
$$

for

The corresponding compact component $\Delta_{C}(\Omega)$ is applicable

$$
\Omega<1 / \mathrm{M},
$$

and use of Eq. (28) in the flyover plane gives:

$\Delta_{C}(\Omega)=10 \cdot \log _{10}\left\{\frac{\operatorname{Min}^{2}\left(\frac{I}{2} \theta\right) I(2 \mathrm{~s} / \mathrm{L})}{4(I+M)(I+M \cos \theta)^{3}}\left|H_{0}^{(I)}\left[\Omega\left(U / U_{3}\right)(\mathrm{s} / \mathrm{L})\right]\right|^{2}\right\}$.

Figures $3,4,5$ illustrate the predicted variations in $\Delta_{\mathrm{C}}$ (dashed curves) and $\Delta_{\mathrm{NC}}$ (dotted) with Strouhal number $\Omega$ for three different radiation directions $\theta$, and for forward flight Mach numbers $M=0.1,0.3,0.5$ respectively. The slot width/length ratio $2 \mathrm{~s} / \mathrm{L}=0.1$ and $\mathrm{U}_{3} / \mathrm{U}$ is taken to be 0.2 . 


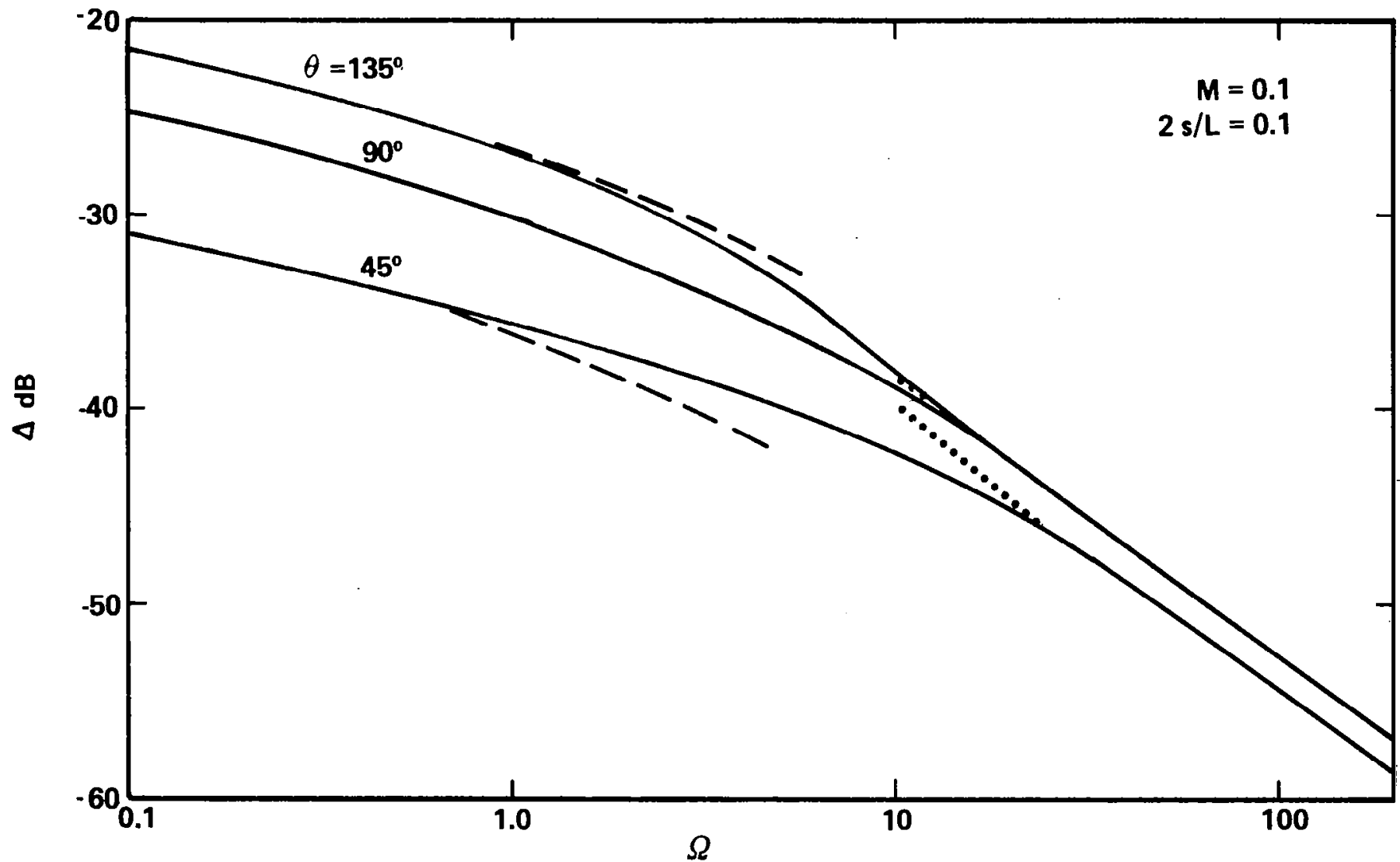

FIG. 3. VARIATION OF $\Delta(\Omega)$ (EQUATION (39)) WITH STROUHAL NUMBER $\Omega=\omega L / U$ IN THE FLYOVER PLANE $\phi=0$, AND FOR $M=0.1, U_{3} / U=0.2,2 \mathrm{~s} / \mathrm{L}=0.1$ : .... NONCOMPACT LIMIT OF EQUATION (40); -..- COMPACT LIMIT, EQUATION (39); POSSIBLE INTERPOLATION CURVE. THE RADIATION ANGLE $\theta$ IS MEASURED FROM THE DOWNSTREAM DIRECTION. 


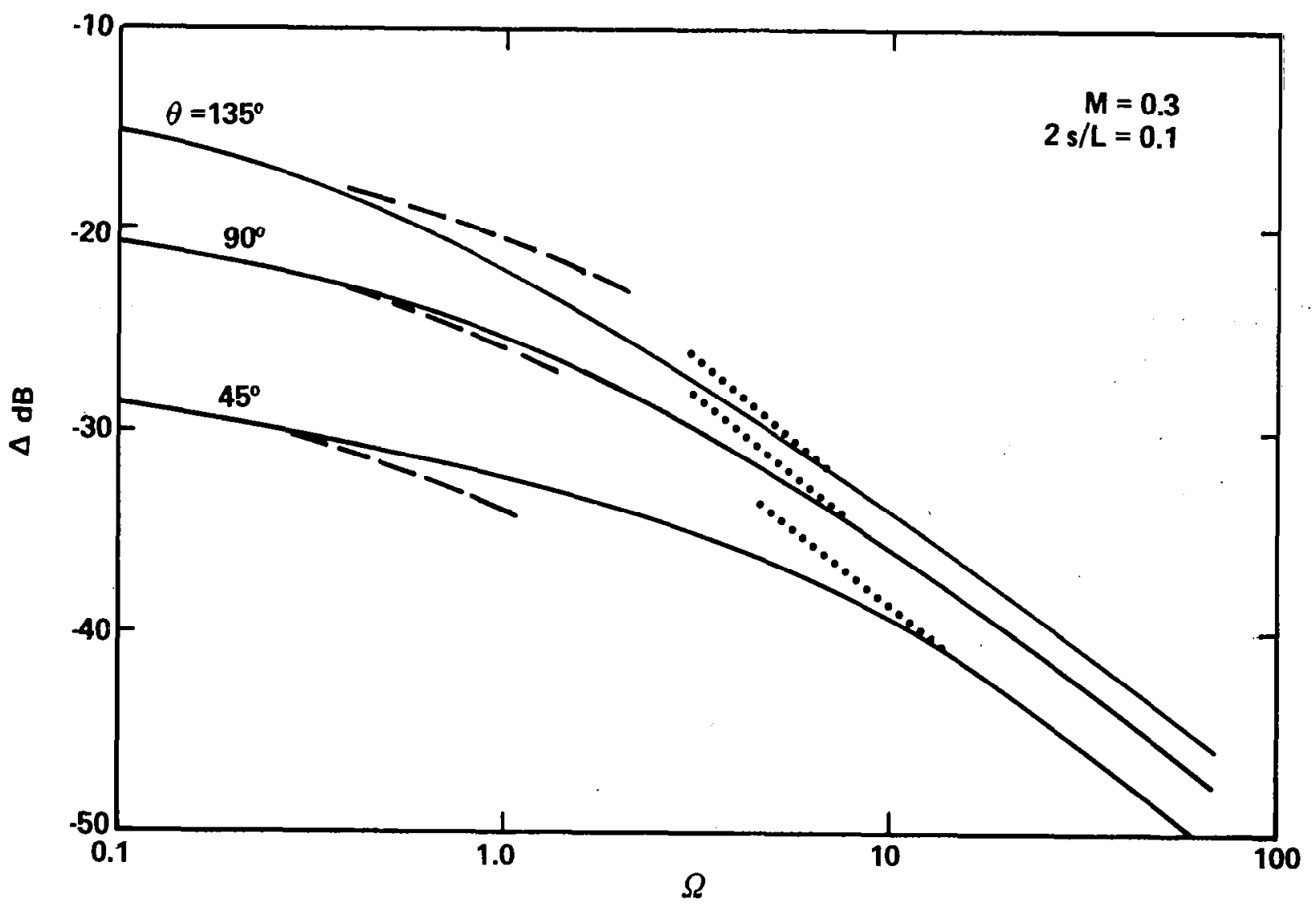

FIG. 4. AS FOR FIG. 3 WITH $M=0.3$. 


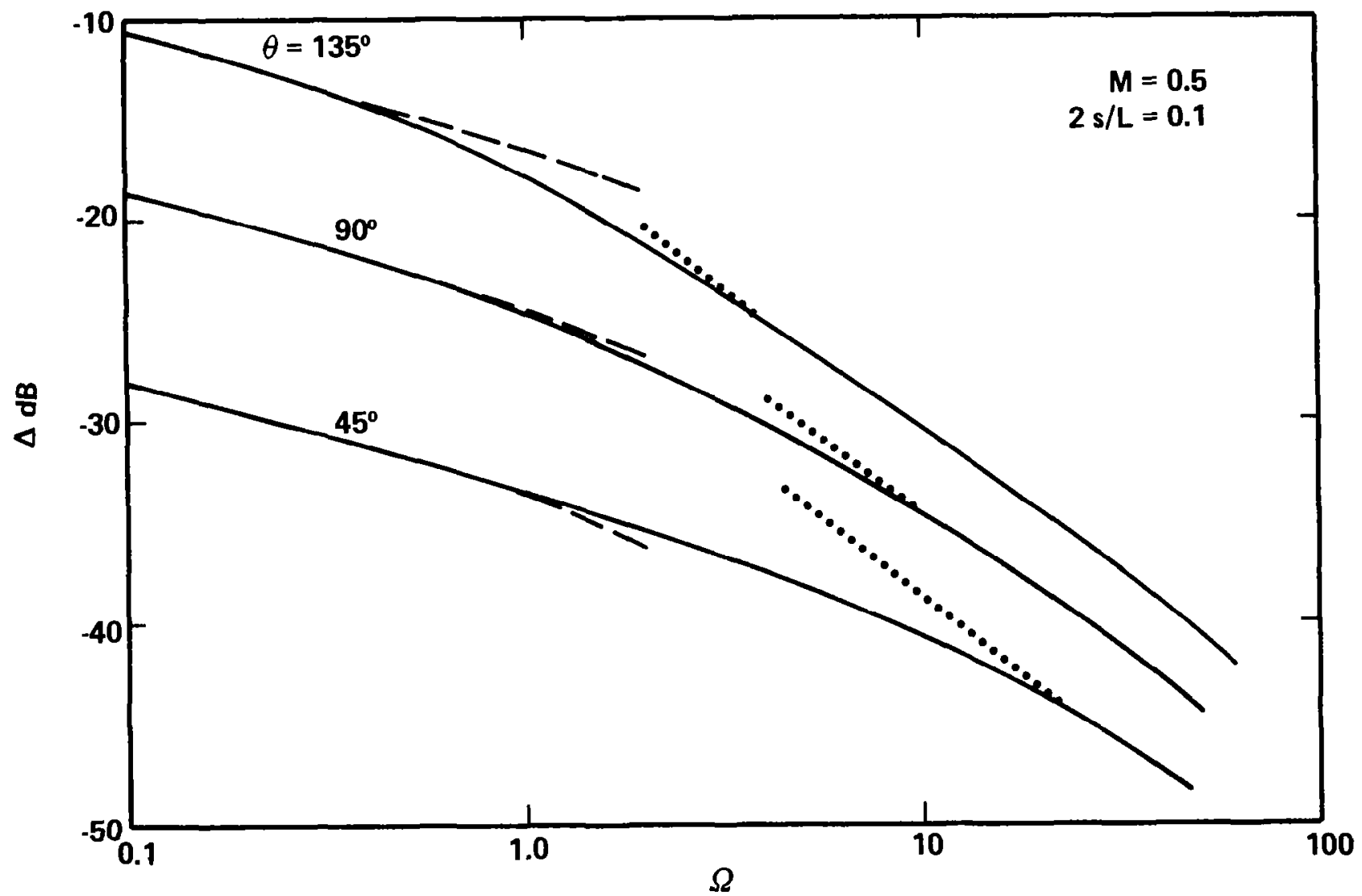

FIG. 5. AS FOR FIG. 3 WITH $M=0.5$. 
The solid curves represent possible interpolative matchings between corresponding asymptotic approximations. There is a degree of arbitrariness in the choice of the interpolation curves, although it is unlikely that the errors involved will exceed about $+3 \mathrm{~dB}$. The divergence of these curves in each figure wit $\bar{h}$ decreasing Strouhal number indicates an accompanying change in effective acoustic source type. At high frequencies the radiation has monopole characteristics, modified by forward flight, and is associated with the unsteady flux through the slot induced by the side-edge turbulence. At low Strouhal numbers, however, that flux is equivalent to a dipole source at the edge of a semi-infinite rigid baffle, and the field shape peaks in the forward arc $\left(\theta=180^{\circ}\right)$ with a null in the downstream direction.

The change in source type with Strouhal number can be illustrated further by means of the field shape plots of Figs. 6, 7, 8, which depict the variation of $\Delta(\Omega)$ with the flyover angle $\theta$ for $\Omega=0.4,4,40$, and for the values of $\mathrm{M}, \mathrm{U} / \mathrm{U}$ and $2 \mathrm{~s} / \mathrm{L}$ used above. The asymptotic formulae for $\Delta_{\mathrm{C}}, \delta_{\mathrm{NC}}$ have been used to draw the curves for $\Omega=0.4,40$. The data points at the intermediate strouhal number $\Omega=4$ have been obtained by making use of interpolation curves of the type shown in Figs. 3-5, and the corresponding field shape (dashed curves in Figs. 6-8) must therefore be regarded as tentative. The plots exhibit the forward flight amplification of sound radiated ahead of the aircraft $\left(\theta>90^{\circ}\right)$, although its magnitude is dependent on the Strouhal number $\Omega$. It is particularly noteworthy that, for nominally equal surface pressure fluctuations $\Phi(\omega)$, the semi-baffled dipole radiation at low Strouhal numbers is typically $10-25$ dB greater than that from the higher frequency monopole sources. This is in contrast to the commonly accepted notions regarding the rank ordering of source strenglh with aeroacoustic source type. The difference arises because the effective monopole/ dipole strength is proportional to the Hankel function $\mathrm{H}(1)\left(\omega_{\mathrm{s}} / \mathrm{U}_{3}\right)$, which is small at high strouhal numbers, but loggarithmically large as $\Omega \rightarrow 0$. This influence of the finite mean width of the side-edge gap is a proximity effect, similar to the so-called installation effects which are known to be important in determining the jet noise of an aircraft [8,9]. Its occurrence in the present investigation emphasizes the importance of taking account of the neighboring undeflected portion of the trailing edge of the airfoil in estimating the radiated sound pressure level. 


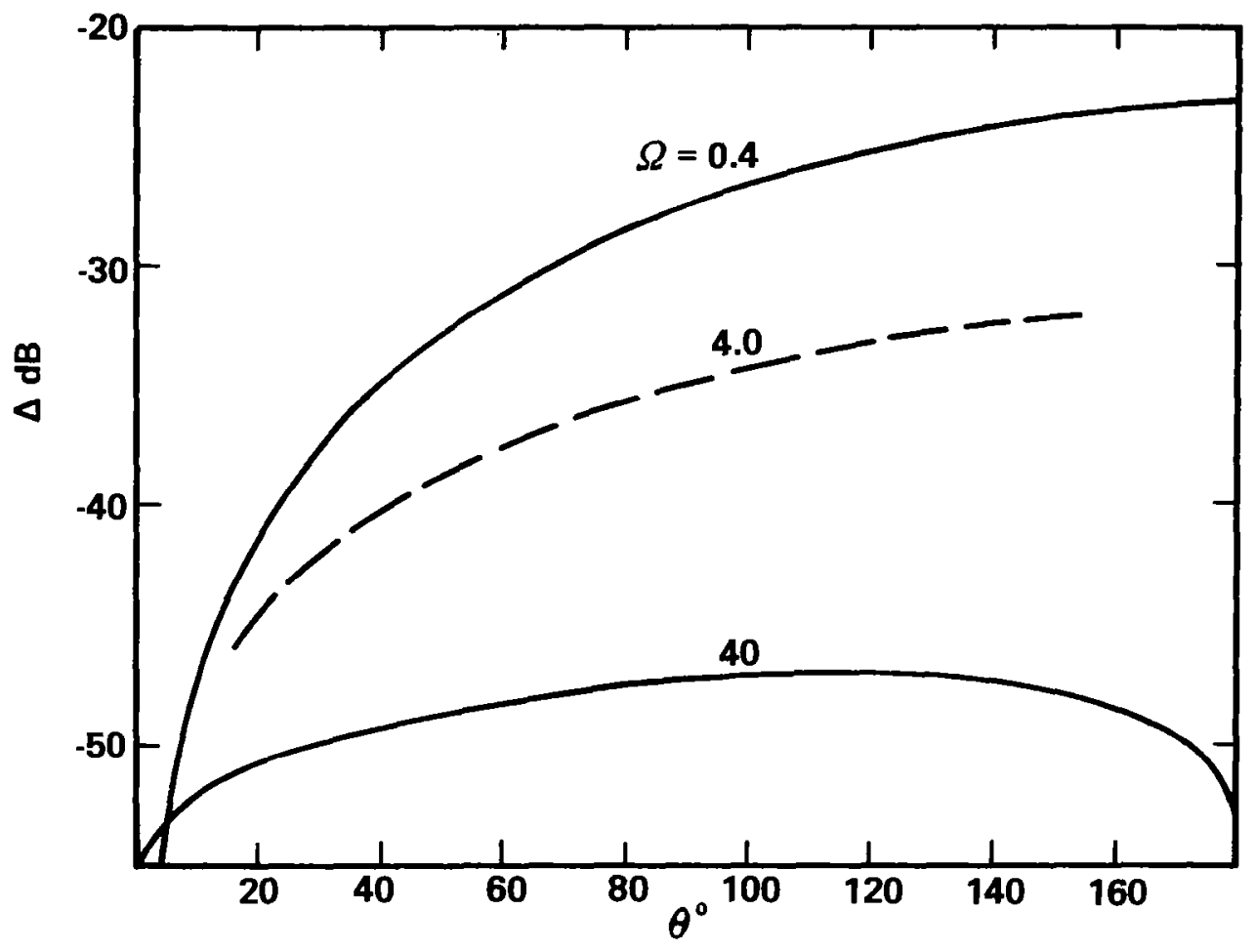

FIG. 6. THE FIELD SHAPE $\triangle(\Omega)$ (EQUATION (39)) IN THE FLYOVER PLANE $\phi=0$, FOR $\Omega=0.4,4,40$, AND FOR $M=0.1, U_{3} / U=0.2,2 \mathrm{~s} / \mathrm{L}=0.1$. THE CURVE FOR $\Omega=4$ HAS BEEN OBTAINED BY USE OF INTERPOLATION CURVES OF THE TYPE SHOWN IN FIGS. $3-5$. 


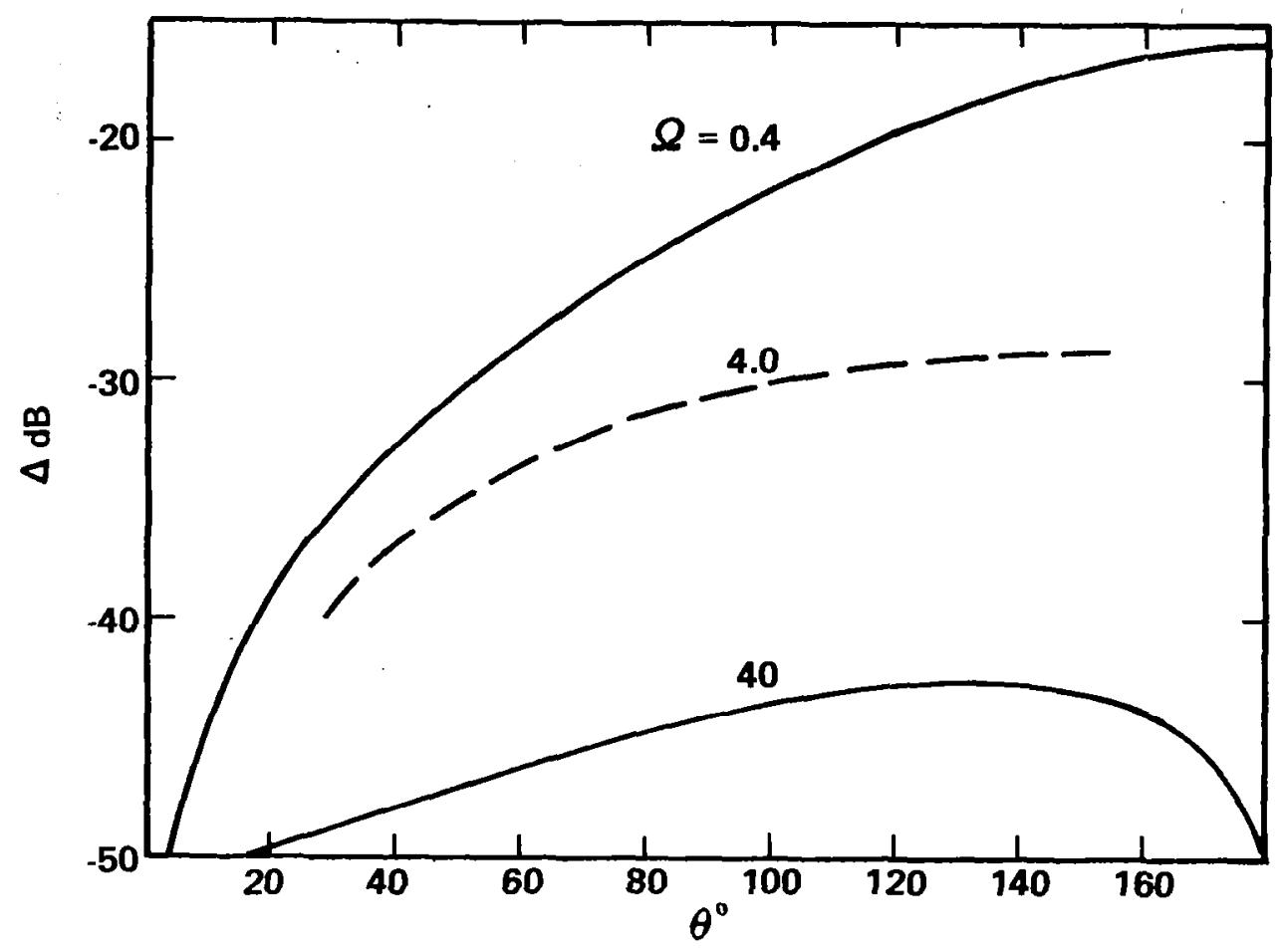

FIG. 7. AS FOR FIG. 6 WITH $M=0.3$. 


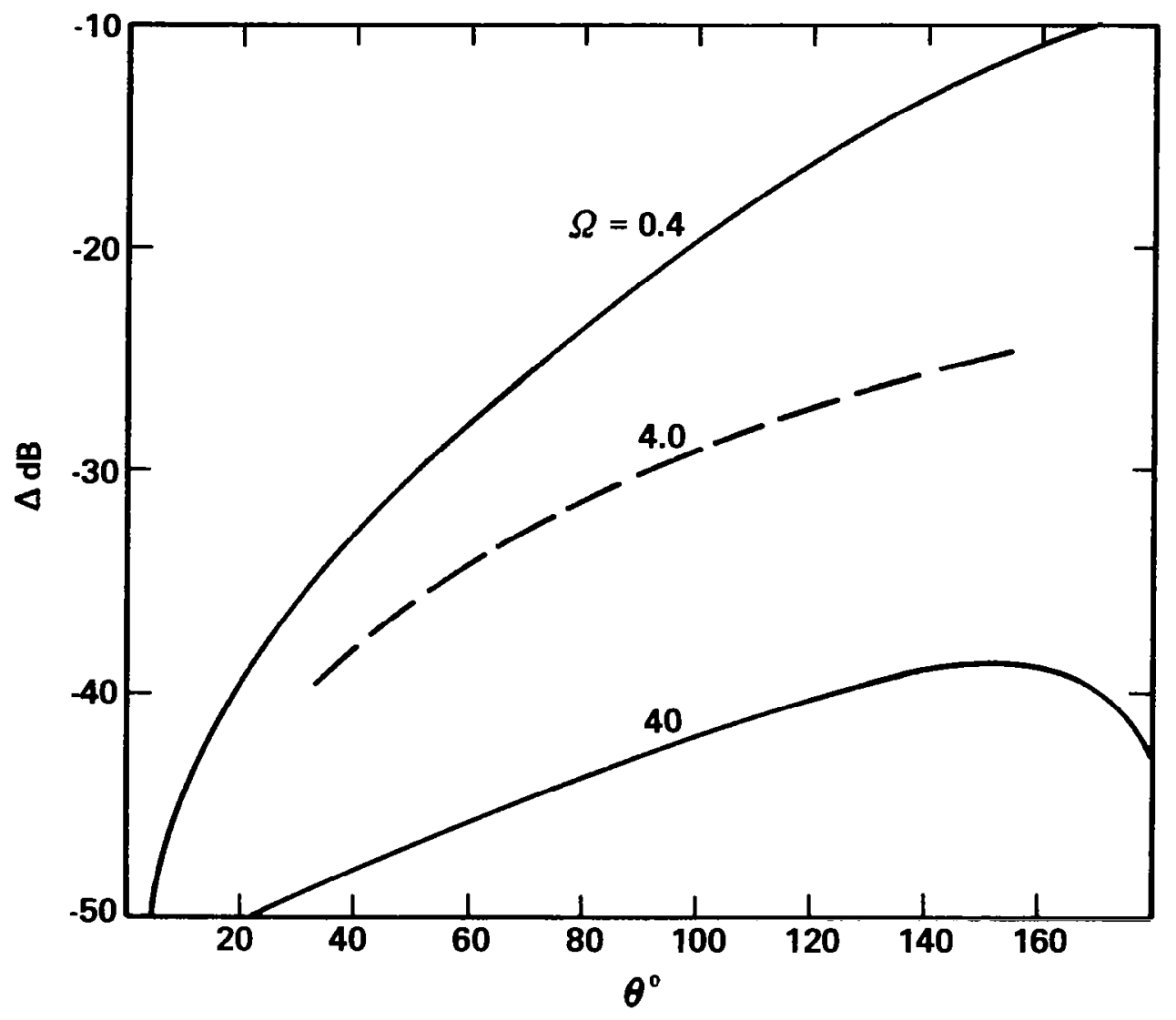

FIG. 8. AS FOR FIG. 6 WITH M $=0.5$. 


\section{COMPARISON WITH EXPERIMENT}

The practical application of the formulae derived above requires a knowledge of the effective surface pressure spectrum $\Phi(\omega)$. Measured values of $\Phi(\omega)$ are not yet available, although some of its important characteristics can be inferred from the very recent experiments of Miller. [20] in the $40 \times 80 \mathrm{ft}^{2}$ wind tunnel at the NASA Ames Research Center. These involved a 1/5-full scale model airfoil of 6.7 meter semi-span with three flaps of span 3.43 meters deployed in tandem. The sound generated at the side edges of the flaps at mean flow velocities up to $79 \mathrm{msec}^{-1}$ was found to exceed that from the midspan region by more than $10 \mathrm{~dB}$, the side edge of the leading flaps being the noisiest. The leading flap was deployed at an angle of $20^{\circ}$, and its geometrical configuration relative to the adjacent portion of the airfoil corresponds rather well with the idealized model of Fig. 1. We shall here assume that the associated values of the surface pressure fluctuations provide a good approximation for estimating the noise generated by the side edges of a single deployed flap.

At low mean flow Mach numbers, it was found that the dominant radiation occurred at sufficiently low frequencies that the chord $\mathrm{L}(\sim 20 \mathrm{cms})$ of the leading flap could be regarded as acoustically compact - the ficld shape resembled that of a semi-baffled dipole. Cross-correlation measurements of the pressure on the flap indicated that these frequencies satisfied: flap thickness $\leqslant 2 \pi \mathrm{U}_{\mathrm{c}} / \omega<\frac{7}{2} \mathrm{~L}$, where $\mathrm{U}_{\mathrm{c}} \sim 0.8 \mathrm{U}$ is the characteristic eddy convection velocity. Side edge turbulent velocity fluctuations were in the range $10-25 \%$ of the mean speed $U$, corresponding to root mean squarc surface pressurc fluctuations $\sqrt{\overline{p_{s}^{2}}}$, say, of order $0.01-0.06$ of the mean dynamic pressure. At the trailing edge of the flap, the root mean square pressure fluctuation $\sqrt{\frac{p_{t e}^{2}}{2}}$, may be identified with that beneath a turbulent boundary layer on a flat plate, and is roughly 0.006 times the mean dynamic pressure [21]. These order of magnitude results can be used to compare the predicted side edge noise with that generated by turbulent flow over the trailing edge of the flap. According to Equations $(78),(80)$ of $[7]$, the acoustic frequency spectrum $S_{t e}(\omega)$ of sound generated by trailing edge turbulence is given at low Mach numbers, and in the flyover plane $(\phi=0)$, by 


$$
S_{t e}(\omega)=\frac{2 M_{c} L_{E_{1}{ }^{l}}}{\pi r_{0}^{2}} \cdot \sin ^{2}\left(\frac{1}{2} \theta\right) \Phi(\omega),
$$

where $\mathrm{L}_{\mathrm{E}}$ is the span of the trailing edge wetted by the turbulent flow, $\ell_{3}$ is the spanwise surface pressure correlation length and, as before $\Phi(\omega)$ denotes the surface pressure frequency spectrum. This result may be combined with the compact approximation formula (36) for the side edge noise in order to estimate the relative importance of the two noise producing mechanisms. To do this, we consider a characteristic midrange frequency $\omega$ for which $2 \pi U_{C} / \omega \approx \frac{1}{4} L$. At small mean flow Mach numbers the side edge generated sound pressure level is predicted to exceed that from the trailing edge by an amount $\triangle S P L$, where

$$
\begin{aligned}
\Delta S P L= & 10 \log _{10}\left\{\pi^{2}\left(\mathrm{~L} / \mathrm{L}_{\mathrm{E}}\right)\left(\ell_{1} / \ell_{3}\right)\left|\mathrm{H}^{(1)}\left[8 \pi(\mathrm{s} / \mathrm{L})\left(\mathrm{U}_{\mathrm{C}} / \mathrm{U}_{3}\right)\right]\right|^{2}\right. \\
& \left.I(2 \mathrm{~s} / \mathrm{L})\left[\overline{\mathrm{p}_{\mathrm{Se}}^{2}} / \overline{\mathrm{p}_{\mathrm{te}}^{2}}\right]\right\}
\end{aligned}
$$

For a flap of chord $L=20 \mathrm{cms}$ deployed at an angle of $20^{\circ}$, the mean value of $2 \mathrm{~s} / \mathrm{I} \simeq 0.17$. Interpolation from Table 1 yields $\mathrm{I}(2 \mathrm{~s} / \mathrm{L}) \simeq 0.053$. Taking $\mathrm{U}_{\mathrm{C}} / \mathrm{U}=0.8$, $\mathrm{U}_{3} / \mathrm{U}=0.3$, then gives

$$
\left|\mathrm{H}_{0}^{(1)}\left[8 \pi(\mathrm{s} / \mathrm{L})\left(\mathrm{U}_{\mathrm{c}} / \mathrm{U}_{3}\right)\right]\right|^{2} \simeq 0.112 \text {. }
$$

Finally, we take $\ell_{1} / \ell_{3}=6.5$. This value is appropriate for turbulent boundary layer flow over a flat plate, but it is probably of the corrcct order in the present case (c.f. [11]). Substituting into Eq. (45), we obtain:

$$
\Delta S P I \simeq 10 \operatorname{Iog}_{10}\left\{0.38\left(\mathrm{~L} / L_{E}\right)\left(\overline{p_{s e}^{2}} / \overline{p_{t e}^{2}}\right)\right\} .
$$

Setting $\mathrm{L}=20 \mathrm{cms}, \mathrm{L}_{\mathrm{E}}=343 \mathrm{cms}$, we deduce that $\triangle \mathrm{SPL}$ Iies in the range $-12 \mathrm{~dB}$ to $3.5 \mathrm{~dB}$, when $\overline{\mathrm{p}_{\mathrm{se}} / \mathrm{p}_{\mathrm{t}}^{2}}$ varies between $2.8,100$ in accordance with the experimental values quoted above. Thus, the noise generated at a single side edge of a part span flap can exceed that generated along the 
whole of the trailing edge of the flap by more than $3 \mathrm{~dB}$. The dimensionless form of Eqs. (45) and (46) indicates that this figure extrapolates to full scale without change.

An alternative expression of this conclusion is obtained by taking $I=L_{E}$ in Eq. (46). This gives a comparison of the effective acoustic source strengths per unit length of side edge/trailing edge. In this case $\triangle S P L$ lies between $0.2-16 \mathrm{~dB}$, which is entirely consistent with the relative magnitude of $10 \mathrm{~dB}$ or more observed by Miller [20].

\section{CONCLUSIONS}

High and low frequency asymptotic formulae have been derived which express the acoustic frequency spectrum in terms of the wavenumber-frequency spectrum of surface pressure fluctuations on the upper surface of a part span flap just in-board of the side edge. From a consideration of approximate versions of these relations it has been concluded that:

1. The radiation has the characteristics of a semi-baffled dipole at small values of the Strouhal number wL/U based on the chord of the flap and the forward flight speed. At high frequencies the radiation pattern resembles that of a monopole source.

2. The degree of Doppler amplification due to forward flight of the aircraft is dependent on the Strouhal number $\omega L / U$, being characterized respectively by 3 , 2 powers of the Doppler factor $1 /(1+M \cos \theta)$ as $\omega \mathrm{L} / \mathrm{U} \rightarrow 0, \infty$. As in the analogous case of noise generated at a trailing edge, there exists a weak dependence on $M$ of the radiation at $90^{\circ}$ to the flight path.

3. The radiation efficiency of side edge noise sources is governed by the value of the mean side edge gap Strouhal number $\omega s / U_{3}$ being larger when $\omega s / U_{3}$ is small.

4. Predictions at intermediate Strouhal numbers can be made by interpolation between the low and high frequency formulae. 
5. Recent experiments of Miller [20] indicate that, at least for small values of the flight Mach number, the dominant radiation from the side edge resembles that of a semi-baffled dipole, the chord of the flap being acoustically compact. Theoretical predictions extrapolated to full scale suggest that the noise generated in this case at a single side edge can exceed that generated along the whole of the trailing edge of the flap by $3 \mathrm{~dB}$. This figure is consistent with Miller's experimental f'indings. 


\section{APPENDIX A. THE GREEN'S FUNCTION FOR A NONCOMPACT SLOT}

It is required to solve the reciprocal, time-harmonic Eq. (7) for $k_{0} L \gg 1$. The width $2 \mathrm{~s}$ of the slot is assumed to be small compared with the acoustic wavelength. It is sufficient to confine attention to the case in which the reciprocal source at $x$ is in the acoustic far field of the slot $(|\underline{x}|>>$ L), and the field point $y$ is in the immediate vicinit $\bar{y}$ of the slot. The resulting form of $G(\underline{x}, \underline{y})$ is appropriate for use in Eq. (11), in which the aeroacoustic sources are at points $\underline{y}$ near the slot and the observer at $x$ is in the far field.

The definitions

$$
\left.\begin{array}{rl}
\left(X_{1}, Y_{1}\right) & =\left(x_{1}, y_{1}\right) /\left(I-M^{2}\right)^{\frac{1}{2}} \\
K_{0} & =k_{0} /\left(I-M^{2}\right)^{\frac{1}{2}}
\end{array}\right\},,
$$

enable Eq. (7) to be reduced to:

$$
\left(\frac{\partial^{2}}{\partial Y_{1}^{2}}+\frac{\partial^{2}}{\partial y_{2}^{2}}+\frac{\partial^{2}}{\partial y_{3}^{2}}+K_{0}^{2}\right) G=\delta\left(X_{1}-Y_{1}\right) \delta\left(x_{2}-y_{2}\right) \delta\left(x_{3}-y_{3}\right)
$$

When the slot is noncompact, such that much of its length is many acoustic wavelengths from either end, the influence of diffraction at the ends can be neglected in calculating the behavior of $G$ near the slot. The problem accordingly becomes locally two-dimensional, and this feature is exploited by introducing the Fourier transform $\bar{\phi}$ of $G$ :

$$
G=\frac{1}{2 \pi} \int_{-\infty}^{\infty} \Phi\left(y_{2}, y_{3}, k\right) e^{i k\left(X_{1}-Y_{1}\right)} d k .
$$


Equation (A3) becomes:

$$
\left(\frac{\partial^{2}}{\partial \mathrm{y}_{2}^{2}}+\frac{\partial^{2}}{\partial \mathrm{y}_{3}^{2}}+\gamma^{2}\right) \bar{\phi}=\delta\left(\mathrm{x}_{2}-\mathrm{y}_{2}\right) \delta\left(\mathrm{x}_{3}-\mathrm{y}_{3}\right),
$$

where

$$
\gamma=\left(K_{0}^{2}-k^{2}\right)^{\frac{1}{2}}
$$

and branch-cuts are taken such that on the real k-axis $\operatorname{Im}(\gamma)>0$ when $|k|>\left|K_{0}\right|$.

The particular solution $\bar{\phi}_{i}$ of (A5) due to the reciprocal source on the right hand side is

$$
\bar{\phi}_{i}=-\frac{\dot{i}}{4} \cdot H_{0}^{(I)}(\gamma|\underline{\bar{y}}-\underline{\bar{x}}|) \text {, }
$$

where $\overline{\mathrm{y}}=\left(\mathrm{y}_{2}, \mathrm{y}_{3}\right), \overline{\mathrm{x}}=\left(\mathrm{x}_{2}, \mathrm{x}_{3}\right)$. Assume for definiteness that $\mathrm{x}_{2}>0$. When the cȳlindrical wave $\bar{\phi}_{i}$ is incident on the slot, and when end-effects are neglected, the diffracted component $\bar{\phi}_{\mathrm{r}}$ of $\bar{\phi}$ in $\mathrm{y}_{2}>0$ may be expressed in the form:

$$
\bar{\phi}_{r}=-\frac{i}{4} \cdot H_{0}^{(I)}\left(\gamma\left|\underline{\bar{y}}-\underline{\bar{x}}^{*}\right|\right)+\beta_{0}^{(I)}(\gamma|\underline{\bar{y}}|),(|\underline{\bar{y}}|>>s) .
$$

The first term on the right hand side is the specularly reflected field in the absence of the slot due to an image source at $\underline{x}^{*}=\left(-x_{2}, x_{3}\right)$. When the wavelength of the incident wave is large compared with the width of the slot, the unsteady flow induced in the slot gives rise to a monopole source of amplitude $\beta$ which is represented by the second term on the right. In $y_{2}<0$ the influence of diffraction at the trailing edge is important only at points close to the edge. The principal component of the field just below the slot is therefore given by

$$
\bar{\phi}=-\beta_{0}^{(I)}(\gamma|\underline{\bar{y}}|),(|\underline{\underline{y}}|>>s),
$$

a monopole equal and opposite in strength to that in Eq. (A8). 
At distances from the slot which are very much smaller than the acoustic wavelength, $\bar{\phi}$ can be represented by the potential of an essentially incompressible fluid, and we can set

$$
\bar{\phi}=A+B \cdot \operatorname{Re}\left\{\ln \left(\frac{z}{s}+\sqrt{\frac{z^{2}}{s^{2}}-1}\right)\right\},
$$

where $A, B$ are constants, and $z=y_{3}+i y_{2}([18], \S 66)$. The values of_ $\beta, A, B$ are determined by matching (AI0) with $\bar{\phi}=\bar{\phi}_{i}+\bar{\phi}_{r}$ given by $(\mathrm{A} 7,8)$ and with $\bar{\phi}$ given by (A9) in their respectiver joint domains of validity.

When the reciprocal source at $\vec{x}$ is in the far field $(\gamma|\vec{x}|>1)$, and $\bar{y}$ is well within a wavelength of the slot, we can use the following asymptotic expressions for $\bar{\phi}$ given by $(A 7,8)$ and (A9)

$$
\begin{aligned}
& \bar{\phi} \simeq 2 Z+\beta\{1+(2 i / \pi) \ln (\underline{\mathrm{C}} \gamma|\underline{\bar{y}}| / 2)\}\left(\mathrm{y}_{2}>0\right), \\
& \bar{\phi} \simeq \quad-\beta\{1+(2 i / \pi) \ln (\underline{\mathrm{C}} \gamma|\underline{\bar{y}}| / 2)\}\left(\mathrm{y}_{2}<0\right),
\end{aligned}
$$

where use has been made of the small argument expansion of the Hankel function $\mathrm{H}_{0}^{(1)}([17], \mathrm{p} .951), \underline{\mathrm{C}}=1.78107 \ldots$ is the Euler-Mascheroni constant, and

$$
z=-\frac{1}{4}(2 i / \pi \gamma|\underline{\bar{x}}|)^{\frac{1}{2}} e^{i \gamma|\underline{\bar{x}}|}
$$

is the asymptotic form of the incident wave $\bar{\phi}_{i}$ as $\gamma|\underline{\bar{x}}| \rightarrow \infty$. Equations (AII), (AI2) are formally valid for

$$
2 \mathrm{~s} \ll|\overline{\mathrm{y}}| \ll 1 /|\gamma|
$$

Note that, although $|\gamma|$ can assume azz positive values, it is only the interval $0<|r|<\left|K_{0}\right|$ which is relevant, wherein the incident disturbance $\phi_{i}$ constitutes a propagating wave ( $\gamma$ real).

(AI0) becomes: 


$$
\bar{\phi} \simeq A+B \operatorname{Bgn}\left(y_{2}\right) \cdot \ln (2|\underline{\bar{y}}| / s) .
$$

Matching this with expressions (All), (AI2) we obtain:

$$
\begin{aligned}
& A=Z \\
& B=\frac{2 i \beta}{\pi}=\frac{-2 i Z}{1+(2 i / \pi) \ln (\underline{c} \gamma s / 4)} .
\end{aligned}
$$

The substitution of these results into Eq. (AI0), and use of (A4) leads to the approximate representation of $G(x, y)$ for points $\mathrm{y}$ well within an acoustic wavelength of the slot. The integration with respect to $k$ may be evaluated by the method of stationary phase when $|\underline{x}| \rightarrow \infty$, yielding

$$
\begin{gathered}
G(\underline{x}, \underline{y}) \simeq \frac{1}{4 \pi R\left(1-M^{2}\right)^{\frac{1}{2}}}\left(1-\frac{2 i R e\left\{\ln \left(\frac{z}{s}+\sqrt{\frac{z^{2}}{s^{2}}-1}\right)\right\}}{\pi\left[1+(2 i / \pi) \ln \left(\underline{C K}_{0} s|\underline{x}| / 4 R\right)\right]}\right) \\
\quad \times \exp \left\{i K_{0}\left[R+M\left(Y_{1}-X_{1}\right)\right]\right\},
\end{gathered}
$$

In which

$$
R=\left\{\left(X_{1}-Y_{1}\right)^{2}+X_{2}^{2}+X_{3}^{2}\right\}^{\frac{1}{2}} .
$$

In the aeroacoustic problem an observer fixed at $\mathrm{x}$ would be stationary with respect to the airfoil, and would therefore be translating at velocity $(-U, 0,0)$ relative to the mean flow. The practical, flyover, case corresponds to an observer at rest relative to the ambient medium, with the aircraft in motion at speed $U$ in the negative $x_{1}$-direction. $T t$ is then convenient to express the radiation in terms of emission time coordinates $(r, \theta, \phi)$, say, which give the observer position relative to the source at the time at which the received sound was generated. The polar angle $\theta$ is measured from the positive direction of the $\mathrm{x}_{1}$-axis, and 


$$
\left.\begin{array}{rl}
x_{1}-y_{1} & =r(M+\cos \theta) \\
x_{2} & =r \cos \phi \sin \theta \\
x_{3} & =r \sin \phi \sin \theta
\end{array}\right\}
$$

It follows that

$$
\left.\begin{array}{c}
R=r(I+M \cos \theta) /\left(I-M^{2}\right)^{\frac{1}{2}} ; R-M\left(X_{1}-Y_{1}\right)=r\left(I-M^{2}\right)^{\frac{1}{2}} ; \\
|\underline{x}| / R=\sin \theta\left(I-\mathbb{M}^{2}\right)^{\frac{1}{2}} /(I+M \cos \theta),
\end{array}\right\}
$$

and therefore that expression (AI6) for $G(\underline{x}, \underline{y})$ assumes the form:

$G(\underline{x}, \underline{y}) \simeq$

$\frac{e^{i k_{0} r}}{4 \pi r(1+M \cos \theta)}\left\{I-\frac{2 i \operatorname{Re}\left\{\ln \left(\frac{z}{s}+\sqrt{\left.\frac{z^{2}}{s^{2}}-I\right)}\right\}\right.}{\pi\left(I+(2 i / \pi) \ln \left[\underline{C} k_{0} s \cdot \sin \theta / 4(1+M \cos \theta)\right]\right)}\right\}$ (A20) 


\section{APPENDIX B. THE GREEN'S FUNCTION FOR A COMPACT SLOT}

An approximate solution of $\mathrm{Eq}$. (A3) will now be obtained in the compact limit for which

$$
k_{0} L /\left(I-M^{2}\right) \equiv K_{0} L^{\prime} \ll I
$$

where

$$
L^{\prime}=L /\left(1-M^{2}\right)^{\frac{1}{2}}
$$

In this case the length scale of variation of $G$ in the neighborhood of the slot is small compared with $1 / K_{0}$. This implies that $K_{0}^{2}$ on the left of $\mathrm{Eq}$. (A3) can be discarded when the field point $\left(\mathrm{Y}_{1}, \mathrm{y}_{2}, \mathrm{y}_{3}\right)$ is close to the slot, where, in a leading approximation, $G$ will satisfy the Laplacc equation.

When the slot is absent the incident and diffracted fields $G_{0}$ due to the reciprocal source on the right of (A3) can be expanded in terms of Bessel and Hankel functions in the form:

$$
\begin{gathered}
G_{0}=\frac{-i}{8 \pi} \sum_{n=0}^{\infty} \int_{-\infty}^{\infty} \varepsilon_{n} \cos \left[\frac{1}{2} n\left(\bar{\theta}_{0}-\pi\right)\right] \cos \left[\frac{1}{2} n(\bar{\theta}-\pi)\right] J_{n / 2}\left(\gamma \rho_{0}\right) H_{n / 2}(1)(\gamma \rho) \\
\quad \times e^{i k\left(x_{3}-y_{3}\right)} d k \quad\left(\rho_{0}<\rho\right)
\end{gathered}
$$

where $\gamma$ is given by Eq. (A6),

$$
\varepsilon_{0}=1, \varepsilon_{n}=2(n=1,2,3, \ldots) \text {, }
$$

and $(\rho, \bar{\theta}),\left(\rho_{0}, \bar{\theta}_{0}\right)$ are polar coordinates defined by

$$
\left(X_{1}, x_{2}\right)=\rho(\cos \bar{\theta}, \sin \bar{\theta}) ;\left(Y_{1}, y_{2}\right)=\rho_{0}\left(\cos \bar{\theta}_{0}, \sin \bar{\theta}_{0}\right),
$$

(see, e.g., Bowman et al. [19] Chapter 8). 
When $K_{0}\left(X_{1}^{2}+x_{2}^{2}+x_{3}^{2}\right)^{\frac{1}{2}}>>1$, and $K_{0}\left(Y_{1}^{2}+y_{2}^{2}+y_{3}^{2}\right)^{\frac{1}{2}}<<1$, the integral in (B2) can be evaluated by the method of stationary phase, leading to:

$$
\begin{gathered}
G_{0} \simeq \frac{-1}{4 \pi R_{0}} \sum_{n=0}^{\infty} \varepsilon_{n} J_{n / 2}\left(K_{0} \rho_{0} \rho / R_{0}\right) \cos \left[\frac{1}{2} n\left(\bar{\theta}_{0}-\pi\right)\right] \cos \left[\frac{1}{2} n(\bar{\theta}-\pi)\right] \\
\times e^{i\left(K_{0} R_{0}-n \pi / 4\right)},
\end{gathered}
$$

where

$$
R_{0}=\left(X_{1}^{2}+x_{2}^{2}+x_{3}^{2}\right)^{\frac{1}{2}} \text {. }
$$

Let $G_{S}$ denote the additional diffracted field in the presence of the slot. This will satisfy Laplace's equation in the vicinity of the slot. At distances from the slot which are large compared with its width 2 s yet small relative to $I / K_{0}$ (i.e., relative to the acoustic wavelength), $G_{S}$ can be represented by the potential of a line source of strength per unit length $\sigma\left(Y_{1}\right)$, say, which occupies the portion $\left(-L^{1}<Y_{1}<0\right)$ of the $Y_{1}$-axis on the upper surface $\left(y_{2}=+0\right)$ of the haltplane, together with a similarly located line sink of equal strength on the lower surface. This can be expressed in terms of the incompressible limit of Macdonald's representation of the Green's function for a rigid half-plane ([19], Chapter 8), from which one finds:

$$
G_{S}=\frac{-\operatorname{sgn}\left(y_{2}\right)}{2 \pi} \int_{-L}^{0} \frac{\sigma(\xi)}{R}\left\{I-\frac{4}{\pi} \cdot \tan ^{-1}\left(\frac{R}{R_{1}+\left(R_{1}^{2}-R^{2}\right)^{\frac{3}{2}}}\right)\right\} d \xi \text {. }
$$

where 


$$
\left.\begin{array}{rl}
R & =\left\{\left(Y_{1}-\xi\right)^{2}+y_{2}^{2}+y_{3}^{2}\right\}^{\frac{1}{2}} \\
R_{1} & =\left\{\left(\rho_{0}-\xi\right)^{2}+y_{3}^{2}\right\}^{\frac{1}{2}}
\end{array}\right\}
$$

The principal branch of $\tan ^{-1}(z)$, which vanishes at $z=0$, is to be taken in ( $B 5)$.

$$
\begin{aligned}
& \text { Equations (B3), (B5) define } \\
& G=G_{0}+G_{S},
\end{aligned}
$$

in those regions whose closest distance from the slot greatly exceeds the slot width $2 \mathrm{~s}$, but is much less than the acoustic wavelength. Provided that $2 s \ll I$, the behavior of $G$ at the slot can be taken in a form analogous to (AI0), namely:

$$
G=A+B\left(Y_{1}\right) \cdot \operatorname{Re}\left\{\ln \left[\frac{z}{S}+\sqrt{\frac{z^{2}}{s^{2}}-I}\right]\right\},\left(-L^{\prime}<Y_{1}<0\right),
$$

where $z=y_{3}+i y_{2}, A$ is constant, and $B\left(Y_{1}\right)$ varies significantly only over distances which are large compared with s.

The local source strength $B\left(Y_{1}\right)$ is determined in terms of $\sigma\left(Y_{1}\right)$ as follows. For fixed $Y_{1}$ in $\left(-L^{\prime}, 0\right)$, and for $|z|>s$ :

$$
G \simeq A+B\left(Y_{1}\right) \operatorname{sgn}\left(y_{2}\right) \ln (2|z| / s)
$$

This is valid in the region

$$
2 \mathrm{~s}<|z|<\mathrm{L}^{\prime}, \quad-\mathrm{L}^{\prime}<\mathrm{Y}_{1}<0 \text {. }
$$

The formal expression (B9) must be equivalent to that given by $(\mathrm{B} 3, \mathrm{~B} 5, \mathrm{~B} 7)$ as $|\mathrm{z}| \rightarrow 0\left(-\mathrm{L}^{\prime}<\mathrm{Y}_{1}<0\right)$ in (B7). In this limit the integral in (B5) exhibits a logarithmic singularity of the type occuring in (B9), and equating the coefficients of the logarithms we find:

$$
\sigma\left(Y_{1}\right) / \pi=B\left(Y_{1}\right)
$$


Equations which determine the values of $A, B\left(Y_{1}\right)$ are obtained by matching the remaining, finite terms in (B7), (B9). We do this numerically by partitioning the interval $-L^{\prime}<\mathrm{Y}<0$ occupied by the slot into $\mathrm{N}$ equal parts, each of width $h=L / N$. The midpoint of the nth partition is at $Y_{1}=Y_{n}$, where

$$
Y_{n}=\left(n-N-\frac{1}{2}\right) h \quad \text {. }
$$

Within the range $\left(Y_{n}-\frac{1}{2} h, Y_{n}+\frac{1}{2} h\right) B\left(Y_{1}\right)$ is taken to be constant and equal to $B_{n}$. A collocation procedure may now be used to obtain $N+1$ equations for $A, B_{n}(n=1,2, \cdots, N)$. Set $Y_{1}=Y_{n}$ in Eqs. (B7), (B9). Take the limit $\left|\frac{\mathrm{y}}{\mid}\right| \equiv|\mathrm{z}| \rightarrow 0$ in (B7) and match the resulting expression with that on the right of (B9). Making use of the identification (B10) we find:

$$
A=-\frac{e^{i K_{0} R_{0}}}{4 \pi R_{0}},
$$

$$
\begin{aligned}
B_{n} I n(2 h / s)+ & \sum_{m=1}^{N} I_{m}^{\frac{1}{2}} B_{m} \int_{Y_{m}-\frac{1}{2} h}^{Y_{m}+\frac{1}{2} h} \frac{d \xi}{\left|Y_{n}-\xi\right|}\left\{\varepsilon_{n m}-\frac{4}{\pi} \cdot \tan ^{-1}\left[\frac{\left|Y_{n}-\xi\right|}{\left(|\xi|^{\frac{1}{2}}+\left|Y_{n}\right|^{\frac{1}{2}}\right)^{2}}\right]\right\} \\
& =-\frac{e^{i K_{0} R_{0}}}{2 \pi R_{0}}\left(2 K_{0} \rho / Y_{n} \mid / i \pi R_{0}\right)^{\frac{1}{2}} \sin \left(\frac{1}{2} \theta\right) \quad(n=1,2, \cdots, N),
\end{aligned}
$$

in which

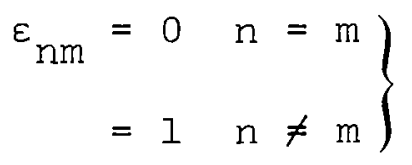

The terms on the right of (BI2), (BI3) correspond to the inner limit $(|\underline{\underline{y}}| \rightarrow 0)$ of $G$, which is given by Eq. (B3). Since $K_{0} \rho_{0} \ll I$ in the vicinity of the slot, only the first two terms in the expansion of $G_{0}$ in terms of $K_{0} \rho_{0}$ have been 
retained. The component of the integral on the left of (BI3) which involves $\varepsilon_{\mathrm{nm}}$ is evaluated analytically, and that involving the inverse tangent is approximated by means of the trapezoidal formula. The system of linear equations for the $B_{n}$ turns out to be stable provided that the logarithm shown explicitly on the left hand side of (BI3) exceeds unity, i.e. for $2 \mathrm{~h} / \mathrm{s} \approx 2.7$.

Set

$$
\lambda=\left|Y_{1}\right| / L=\left|y_{1}\right| / L,
$$

and introduce the dimensionless function $m(\lambda)$ defined in terms of $B\left(Y_{1}\right)$ by

$$
B\left(Y_{1}\right)=-\frac{m(\lambda)}{2 \pi R_{0}}\left(2 K_{0} L^{\prime} \rho / i \pi R_{0}\right)^{\frac{1}{2}} \sin \left(\frac{1}{2} \bar{\theta}\right) e^{i K_{0} R_{0}} .
$$

The corresponding discrete values $m_{n}$ satisfy Eqs. (BI3) with the right hand sides replaced by $\left(\left|\frac{n}{n}\right| / L\right)^{\frac{1}{2}}=\left\{\left(N-n+\frac{1}{2}\right) / N\right\}^{\frac{1}{2}}$, $(n=1,2, \cdots, N)$. The local source strength $B\left(Y_{1}\right)$ and its dimensionless version $\mathrm{m}(\lambda)$ are dependent on the slot width/ length ratio $2 \mathrm{~s} / \mathrm{L}$, and the computed variations of $\mathrm{m}(\lambda) \quad(0<\lambda<1)$ for $2 \mathrm{~s} / \mathrm{L}=0.02,0.05,0.1$ are depicted in Fig. 2 .

Substituting from (B12), (B16) into the local representation (B8) of $G$, using the definition (A2), and expressing the final result in terms of the emission time coordinates (A18), we obtain, finally,

$$
\begin{aligned}
G(\underline{x}, \underline{y}) \simeq & \frac{e^{i k_{0} r_{0}}}{4 \pi r_{0}(I+M \cos \theta)}\{I \\
& +2 m\left(\left|y_{1}\right| / L\right) \sin \left(\frac{1}{2} \bar{\theta}\right)\left[2 k_{0} L\left(\rho / R_{0}\right) / i \pi\left(1-M^{2}\right)\right]^{\frac{1}{2}} \times \\
& \left.\times \operatorname{Re}\left(\ln \left[\frac{z}{s}+\sqrt{\frac{z^{2}}{s^{2}}-1}\right]\right)\right\},
\end{aligned}
$$


in which

$$
\rho / R_{0}=\left\{(M+\cos \theta)^{2}+\left(1-M^{2}\right) \cos ^{2} \phi \sin ^{2} \theta\right\}^{\frac{1}{2}} /(1+M \cos \theta),
$$

and

$\sin \left(\frac{1}{2} \bar{\theta}\right)=\operatorname{sgn}\left(x_{2}\right)\left\{\frac{1}{2}\left(1-(M+\cos \theta) /\left\{(M+\cos \theta)^{2}+\left(1-M^{2}\right) \cos ^{2} \phi \sin ^{2} \theta\right\}^{\frac{1}{2}}\right)\right\}^{\frac{1}{2}}$

- (B19)

Equation (B17) gives the compact form of the Green's function for points $\underset{\sim}{\mathrm{y}}$ in the immediate vicinity of the slot. 


\section{REFERENCES}

1. M.R. Fink and R.H. Schlinker 1979 NASA CR-3110. Air frame noise component interaction studies.

2. W.F. Ahtye, W.R. Miller, and W.C. Meecham 1979 AIAA Paper 79-0667. Wing and flap noise measured by near and far-field cross-correlation techniques.

3. J.M. Kendall and W.F. Ahtye 1980 AIAA Paper 80-0035. Noise generation by a lifting wing/flap combination at Reynolds numbers to $2.8 \times 10^{6}$.

4. G.J. Healy 1974 NASA CR-2377. Measurement and analysis of aircraft far-field aerodynamic noise.

5. J.C. Hardin 1980 AIAA J. 18, 549-552. Noise radiation from the side edges of flaps.

6. J.E. Ffowcs Williams and L.H. Hall $1970 \mathrm{~J}$. Fluid Mech. 40, 657-670. Aerodynamic sound generation by turbulent flow in the vicinity of a scattering half-plane.

7. M.S. Howe $1978 \mathrm{~J}$. Sound Vib. 61, 437-465. A review of the theory of trailing edge noise.

8. W.D. Bryce 1979 AIAA Paper 79-0648. Experiments concerning the anomalous behavior of aerodynamic exhaust noise in flight.

9. N.N. Reddy and H.K. Tanna 1980 AIAA Paper 80-1044. Installation effects on jet noise in flight.

10. N.A. Chigier and V.R. Corsiglia 1971 NASA Tech. Memo. TM X-62087. Tip vortices-Velocity distributions.

11. K.L. Chandiramani 1974 J. Acoust. Soc. Am. 55, 19-29. Diffraction of evanescent waves with applications to aerodynamically scattered sound and radiation from unbaffled plates.

12. D.M. Chase $1972 \mathrm{~J}$. Acoust. Soc. Am. 52, 101I-1023. Sound radiated by turbulent flow off a rigid half-plane as obtained from a wavevector spectrum of hydrodynamic pressure. 
13. D.M. Chase 1975 AIAA J. 13, 1041-1047. Noise radiated from an edge in turbulent flow.

14. T.F. Brooks and T.H. Hodgson 1980 ATAA Paper 80-0977. Prediction and comparison of trailing edge noise using measured surface pressures.

15. M.S. Howe 1975 J. Fluid Mech. 67, 597-610. The generation of sound by aerodynamic sources in an inhomogeneous steady flow.

16. J.A. Stratton 1941 Eiectromagnetic Theory. New York, McGraw-Hill.

17. I.S. Gradshteyn and I.M. Ryzhik 1980 Table of Integrals, Series and Products. Now York, Academic.

18. H. Lamb 1932 Hydrodynamics (6th edn.) Cambridge University Press.

19. J.J. Bowman, T.B.A. Senior, and P.L.E. Uslenghi 1969. Electromagnetic and acoustic scattering by simple shapes. Amsterdam, North-Holland.

20. W.R. Miller 1980. Flap noise characteristics measured by pressure cross-correlation techniques. Thesis, Uriversity of California, Los Angeles.

21. W.W. Willmarth 1975 An. Rev. Fluid Mech. 7, 13-38. Pressure fluctuations beneath turbulent boundary layers. 


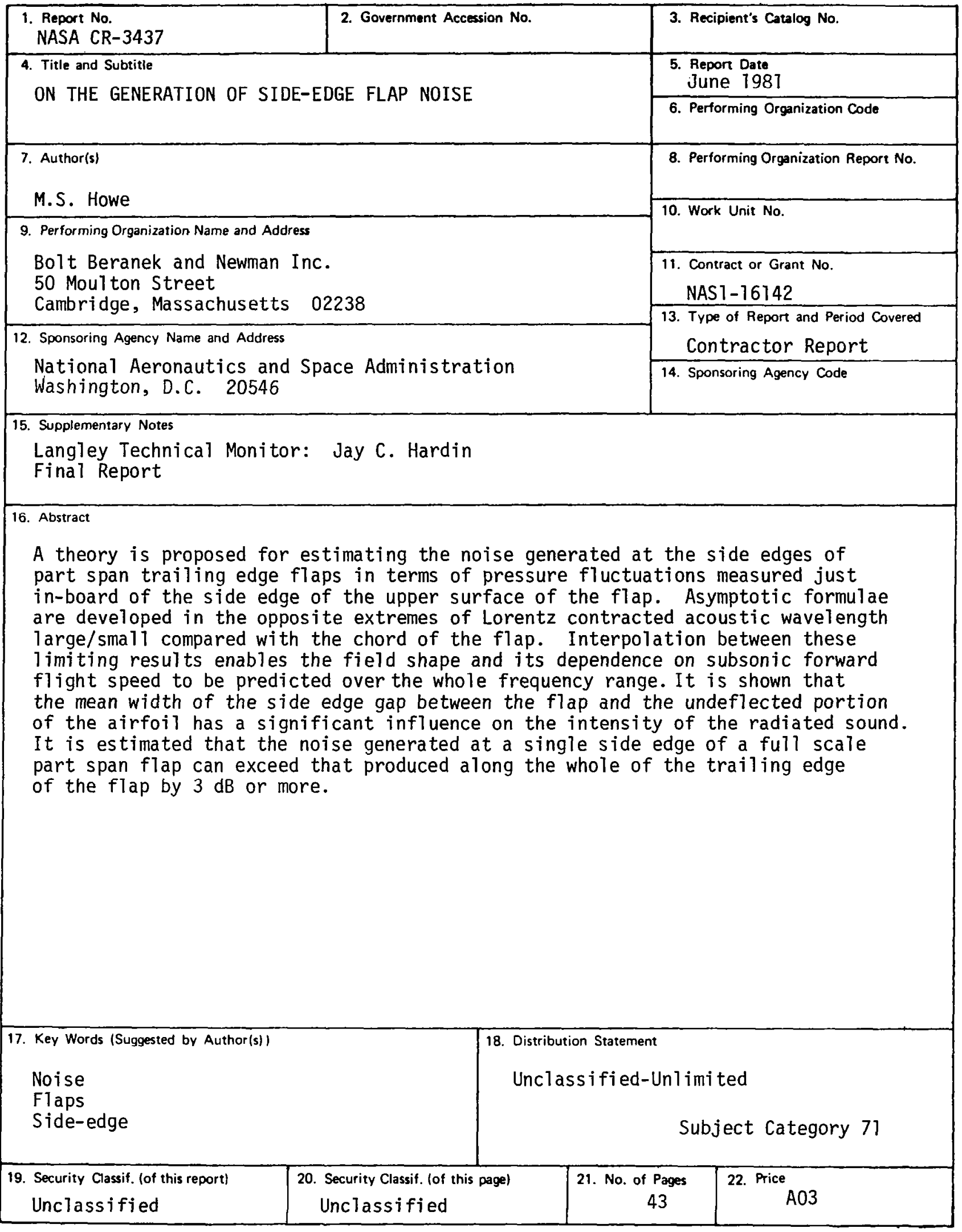

For sale by the National Technical Information Service. Springfield. Virginia 22161 